ALEA, Lat. Am. J. Probab. Math. Stat. 15, 1401-1429 (2018)

DOI: $10.30757 /$ ALEA.v15-52

\title{
Generalized operator-scaling random ball model
}

\section{Hermine Biermé, Olivier Durieu and Yizao Wang}

Laboratoire de Mathématiques et Applications, UMR CNRS 7348

Université de Poitiers, Boulevard Marie et Pierre Curie

86962 Futuroscope Chasseneuil Cedex, France.

E-mail address: hermine.bierme@math.univ-poitiers.fr

Institut Denis Poisson, UMR CNRS 7013

Université de Tours, Parc de Grandmont

37200 Tours, France.

E-mail address: olivier.durieu@univ-tours.fr

Department of Mathematical Sciences

University of Cincinnati, 2815 Commons Way, ML-0025

Cincinnati, OH, 45221-0025, United States.

E-mail address: yizao.wang@uc.edu

Abstract. This article introduces the operator-scaling random ball model, generalizing the isotropic random ball models investigated recently in the literature to anisotropic setup. The model is introduced as a generalized random field and results on weak convergence are established in the space of tempered distributions.

\section{Introduction}

In the past ten years, random ball models have appeared as a simple and yet flexible class of random fields that characterize various types of spatial dependence structures, see Kaj et al. (2007); Breton and Dombry (2009); Biermé et al. (2010); Breton and Dombry (2011); Görgens and Kaj (2014); Gobard (2015); Breton and Gobard (2015); Biermé and Estrade (2006); Pilipauskaite and Surgailis (2016). In particular, in several regimes, their scaling limits are self-similar and with longrange dependence, see Samorodnitsky (2016); Pipiras and Taqqu (2017); Beran et al. (2013). Such properties are desirable when modeling various real world phenomena and thus such results have a broad range of applications.

Received by the editors July 3rd, 2018; accepted October 2nd, 2018.

2010 Mathematics Subject Classification. Primary, 60F05, 60G60; secondary, 60G52.

Key words and phrases. Operator-scaling, stable random field, limit theorem, anisotropic random field, random ball model, generalized random field.

YW's research was supported in part by NSA grant H98230-16-1-0322, Army Research Laboratory grant W911NF-17-1-0006, and Charles Phelps Taft Research Center at University of Cincinnati. 
In words, a random ball model consists in a collection of random balls in $\mathbb{R}^{d}$ with locations following a homogeneous Poisson point process and with independent and identically distributed random radius and weights. Thus, each realization of random balls on the space can be naturally viewed as a linear functional on an appropriate space of test functions. Asymptotic behaviors are then of interest, when all the balls are simultaneously rescaled by a parameter $\rho$, and at the same time the intensity of balls also changes with respect to $\rho$. Under mild assumption on the distribution of the radius, limit theorems can be established for $\rho \rightarrow 0$ or $\rho \rightarrow \infty$, corresponding to the zoom-out or zoom-in cases respectively. In both cases, the qualitative behavior of the limit random fields, whether exhibiting spatial dependence or not, depends on whether the random balls are dense or sparse in the limit, in certain sense to be specified below.

The random ball models can be viewed as generalizations of certain onedimensional models based on Poisson point processes that appeared in the study of Internet traffics, see for example Mikosch et al. (2002); Kaj and Taqqu (2008) and references therein. However, the extension to high dimensions presents new technical challenges, and should not be viewed as simple generalization of the onedimensional results. In particular, the developments until now have two main limitations. First, results so far in the literature focus on isotropic random ball models (except for Pilipauskaite and Surgailis, 2016). That is, the random fields have the same distribution in each different direction. This feature, from the application point of view, makes the model much less attractive. Second, the tightness of the scaled random fields is difficult to establish. Usually random ball models are defined as a random field $\{X(\mu)\}_{\mu \in \mathcal{M}}$ indexed by a family of measures $\mathcal{M}$ on $\mathbb{R}^{d}$. The tightness of such random fields, after appropriate normalizations, is only established for very restricted classes of $\mathcal{M}$, see e.g. Breton and Gobard (2015); Breton and Dombry (2011).

The goal of this paper is to establish limit theorems for a general class of random ball models, and to remove the aforementioned two limitations.

First, we provide a general framework of random ball models exhibiting anisotropic features and hence include all previously considered ones as special cases. It is now well understood that a natural generalization of notion of selfsimilarity, widely used in stochastic processes and time series, is the so-called operator-scaling property for random fields introduced by Biermé et al. (2007). A random field $\left\{Z_{t}\right\}_{t \in \mathbb{R}^{d}}$ is said to be $(E, H)$-operator-scaling, if

$$
\left\{Z_{c^{E} t}\right\}_{t \in \mathbb{R}^{d}} \stackrel{\mathrm{d}}{=} c^{H}\left\{Z_{t}\right\}_{t \in \mathbb{R}^{d}}, \text { for all } c>0,
$$

where $E$ is an appropriate $d \times d$ matrix, $c^{E}:=\sum_{k=0}^{\infty}(E \log c)^{k} / k$ ! is also a matrix, and $H>0$. Taking $E$ to be the identity matrix, the above says that the random field $Z$ is self-similar. The motivation of allowing general matrix $E$ is to generalize this notion to anisotropic random fields. Such random fields are often of practical importance in various applications, and they also present theoretical challenges. Families of anisotropic random fields are known, and path properties have been investigated. See for example Biermé and Lacaux (2009); Li et al. (2015); Meerschaert et al. (2013); Xiao (2009). At the same time, the development of limit theorems for anisotropic random fields is still at an early stage. For some recent results, see for example Biermé et al. (2017); Li and Xiao (2012); Wang (2014); Puplinskaite and Surgailis (2015); Lavancier (2007); Durieu and Wang (2018+); Shen 
and Wang (2017). In this article, we also consider more general random sets than balls, precisely sets of finite perimeter.

Second, we view the random ball models as distribution-valued random elements, also known as generalized random fields, and establish weak convergence in the space of tempered distributions. A complete description of self-similar generalized Gaussian random fields was obtained by Dobrushin (1979) and allows to obtain essentially all Gaussian, translation- and rotation-invariant, $H$-self-similar generalized random field as scaling limits of a random balls model, see Biermé et al. (2010). Beyond the Gaussian framework, generalized Lévy random field, including stable generalized random field have been investigated by Unser and Tafti (2014), where they are named as sparse stochastic processes. Distribution-valued random variables and stochastic processes are already widely used to describe fluctuations of empirical measures of complex particle systems, including notably interacting particle systems (Kipnis and Landim, 1999) and branching particle systems (Holley and Stroock, 1978; Kipnis and Landim, 1999; Bojdecki et al., 2007; Li and Xiao, 2012), just to mention a few.

The paper is organized as follows. Section 2 presents background on generalized random fields, the precise definition of the random ball model, and the four regimes of convergence that we investigate. The limit theorems are stated in Section 3, while their proofs are postponed in Section 6. In Section 4, we study statistical properties of the limit random fields. To conclude, a pointwise representation is obtained in Section 5 and some illustrations are given in the appendix.

Throughout, $C$ stands for real constants that may change values from line to line. Without ambiguity, for $x \in \mathbb{R}^{d},|x|$ denotes its Euclidean norm. We write $a \vee b=\max (a, b)$ and $a \wedge b=\min (a, b)$ for $a, b \in \mathbb{R}$.

\section{Background and definitions}

2.1. Generalized random fields. The standard references for generalized random fields include notably Gel'fand and Vilenkin (1964); Gel'fand and Shilov (1964); Dobrushin (1979); Kallianpur and Xiong (1995); Fernique (1967). In words, these fields are defined as random variables with values in a space of distributions (or generalized functions). To this end we consider the Schwartz space $\mathcal{S}\left(\mathbb{R}^{d}\right)$ of all real-valued infinitely differentiable rapidly decreasing functions on $\mathbb{R}^{d}$, and $\mathcal{S}^{\prime}\left(\mathbb{R}^{d}\right)$ its topological dual, the space of tempered distribution. As usual $\mathcal{S}\left(\mathbb{R}^{d}\right)$ is equipped with the topology that corresponds to the following notion of convergence: $f_{n} \rightarrow f$ if and only if for all $N \in \mathbb{N}:=\{0,1,2, \ldots\}$ and $j=\left(j_{1}, \ldots, j_{d}\right) \in \mathbb{N}^{d}$

$$
\left\|f_{n}-f\right\|_{N, j}:=\sup _{z \in \mathbb{R}^{d}}(1+|z|)^{N}\left|D^{j}\left(f_{n}-f\right)(z)\right| \rightarrow 0, \text { as } n \rightarrow \infty,
$$

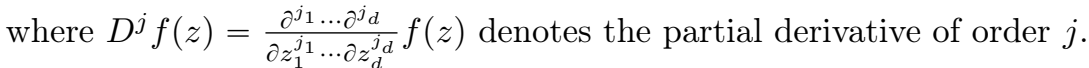

We will actually also consider the space

$$
\mathcal{S}_{1}\left(\mathbb{R}^{d}\right):=\left\{f \in \mathcal{S}\left(\mathbb{R}^{d}\right) ; \int_{\mathbb{R}^{d}} f(z) d z=0\right\} .
$$

Note that $\mathcal{S}_{1}\left(\mathbb{R}^{d}\right)=\operatorname{span}\left\{D^{j} f ; f \in \mathcal{S}\left(\mathbb{R}^{d}\right), j \in\{0,1\}^{d}, j_{1}+\cdots+j_{d}=1\right\}$. For convenience, we also write $\mathcal{S}_{0}\left(\mathbb{R}^{d}\right)=\mathcal{S}\left(\mathbb{R}^{d}\right)$ and thus we will be able to use $\mathcal{S}_{n}\left(\mathbb{R}^{d}\right)$ for $n \in\{0,1\}$ in the sequel. We denote by $\mathcal{S}_{n}^{\prime}\left(\mathbb{R}^{d}\right)$ the topological dual of $\mathcal{S}_{n}\left(\mathbb{R}^{d}\right)$ 
and by $(\cdot, \cdot)$ the duality bracket. We usually consider two distinct topologies on $\mathcal{S}_{n}^{\prime}\left(\mathbb{R}^{d}\right)$. The strong topology is induced by the family of semi-norms

$$
q_{B}(\cdot)=\sup _{f \in B}|(\cdot, f)|, \quad B \text { bounded in } \mathcal{S}_{n}\left(\mathbb{R}^{d}\right) .
$$

The weak topology on $\mathcal{S}_{n}^{\prime}\left(\mathbb{R}^{d}\right)$ is the topology induced by the family of semi-norms $|(\cdot, f)|, f \in \mathcal{S}_{n}\left(\mathbb{R}^{d}\right)$. A first remark is that both topologies generate the same Borel $\sigma$-field denoted by $\mathcal{B}\left(\mathcal{S}_{n}^{\prime}\left(\mathbb{R}^{d}\right)\right)$, see Biermé et al. (2017).

A generalized random field is an $\mathcal{S}_{n}^{\prime}\left(\mathbb{R}^{d}\right)$-valued random variable, that is a measurable mapping $X$ from a probability space $(\Omega, \mathcal{A}, \mathbb{P})$ to $\left(\mathcal{S}_{n}^{\prime}\left(\mathbb{R}^{d}\right), \mathcal{B}\left(\mathcal{S}_{n}^{\prime}\left(\mathbb{R}^{d}\right)\right)\right)$. For such a generalized random field $X$, we let its evaluation at $f \in \mathcal{S}_{n}\left(\mathbb{R}^{d}\right)$ be denoted by $X(f)$, which is a real random variable on the same probability space.

The law of a generalized random field $X$ is uniquely determined by its characteristic functional

$$
\mathcal{L}_{X}(f):=\int_{\Omega} e^{i X(f)} d \mathbb{P}, \quad f \in \mathcal{S}_{n}\left(\mathbb{R}^{d}\right) .
$$

Further, $X$ induces a family of random variables $X(f)$ on $(\Omega, \mathcal{A})$ indexed by $f \in$ $\mathcal{S}_{n}\left(\mathbb{R}^{d}\right)$, with characteristic functions given by

$$
\mathbb{E}\left(e^{i t X(f)}\right)=\int_{\Omega} e^{i t X(f)} d \mathbb{P}=\mathcal{L}_{X}(t f), \quad t \in \mathbb{R} .
$$

By linearity, the finite-dimensional distributions of $X$ are simply obtained with

$$
\mathcal{L}_{X}\left(a_{1} f_{1}+\cdots+a_{k} f_{k}\right)=\mathbb{E}\left(e^{i\left[a_{1} X\left(f_{1}\right)+\cdots+a_{k} X\left(f_{k}\right)\right]}\right),
$$

for all $k \geqslant 1, a_{1}, \ldots, a_{k} \in \mathbb{R}$ and $f_{1}, \ldots, f_{k} \in \mathcal{S}_{n}\left(\mathbb{R}^{d}\right)$.

In practice, however, given a family of real random variables $\{X(f)\}_{f \in \mathcal{S}_{n}\left(\mathbb{R}^{d}\right)}$ on a probability space $(\Omega, \mathcal{A}, \mathbb{P})$ satisfying

$$
X(a f+b g)=a X(f)+b X(g) \text { a.s. for all } a, b \in \mathbb{R}, f, g \in \mathcal{S}_{n}\left(\mathbb{R}^{d}\right),
$$

a priori it is not clear whether a corresponding $\mathcal{S}_{n}^{\prime}\left(\mathbb{R}^{d}\right)$-valued random variable exists. When this can be achieved, namely if there exists an $\mathcal{S}_{n}^{\prime}\left(\mathbb{R}^{d}\right)$-valued random variable $\tilde{X}$, possibly defined on another probability space $(\tilde{\Omega}, \tilde{\mathcal{A}}, \tilde{\mathbb{P}})$, such that for all $k \geqslant 1, f_{1}, \ldots, f_{k} \in \mathcal{S}_{n}\left(\mathbb{R}^{d}\right), A_{1}, \ldots, A_{k} \in \mathcal{B}(\mathbb{R})$,

$$
\mathbb{P}\left(X\left(f_{1}\right) \in A_{1}, \ldots, X\left(f_{k}\right) \in A_{k}\right)=\tilde{\mathbb{P}}\left(\tilde{X}\left(f_{1}\right) \in A_{1}, \ldots, \tilde{X}\left(f_{k}\right) \in A_{k}\right),
$$

we say that $\tilde{X}$ is a version of $X=\{X(f)\}_{f \in \mathcal{S}_{n}\left(\mathbb{R}^{d}\right)}$, as in Definition 9.1.1 of Samorodnitsky and Taqqu (1994). Let us quote that this notion is weaker than the notion of regularization from Itô (1983). Actually, a regularization $\tilde{X}$ of $X$ should be defined on the same probability space $(\Omega, \mathcal{A}, \mathbb{P})$ than $X$ and satisfies $\tilde{X}(f)=X(f)$ a.s. for all $f \in \mathcal{S}_{n}\left(\mathbb{R}^{d}\right)$. However, when we deal with convergence in law for most of the part of the paper, the notion of version is enough for our purpose: once the existence of a version is proved, it suffices to work with the characteristic functionals of the original individual random variables. At only a few occasions we shall establish results in the stronger notion of regularization.

We recall below two fundamental theorems when working with limit theorems of generalized random fields, both based on characteristic functionals. The following theorem is a direct consequence of Minlos-Bochner's theorem, see Corollary 2.2 in Biermé et al. (2017). 
Theorem 2.1. Let $X=\{X(f)\}_{f \in \mathcal{S}_{n}\left(\mathbb{R}^{d}\right)}$ be a collection of real random variables on $(\Omega, \mathcal{A}, \mathbb{P})$ satisfying $(2.1)$. If $\mathcal{L}_{X}: \mathcal{S}_{n}\left(\mathbb{R}^{d}\right) \rightarrow \mathbb{C}$ is continuous then $X$ admits a version that is an $\mathcal{S}_{n}^{\prime}\left(\mathbb{R}^{d}\right)$-valued random variable.

Recall that a sequence of generalized random fields $\left\{X_{m}\right\}_{m \geqslant 1}$ converges in distribution to $X$, denoted by $X_{m} \Rightarrow X$, in $\mathcal{S}_{n}^{\prime}\left(\mathbb{R}^{d}\right)$ given the strong topology if for all $\varphi: \mathcal{S}_{n}^{\prime}\left(\mathbb{R}^{d}\right) \rightarrow \mathbb{R}$ continuous for the strong topology and bounded,

$$
\int_{\mathcal{S}_{n}^{\prime}\left(\mathbb{R}^{d}\right)} \varphi(u) d \mathbb{P}_{X_{m}}(u) \underset{m \rightarrow \infty}{\longrightarrow} \int_{\mathcal{S}_{n}^{\prime}\left(\mathbb{R}^{d}\right)} \varphi(u) d \mathbb{P}_{X}(u) .
$$

Similarly, $X_{m} \Rightarrow X$ in $\mathcal{S}_{n}^{\prime}\left(\mathbb{R}^{d}\right)$ given the weak topology, if the above holds for all $\varphi: \mathcal{S}_{n}^{\prime}\left(\mathbb{R}^{d}\right) \rightarrow \mathbb{R}$ that is bounded and continuous with respect to the weak topology. As a consequence of Lévy's continuity theorem (Theorem 2.3 in Biermé et al., 2017), we can state the following result, see Corollary 2.4 in Biermé et al. (2017).

Theorem 2.2. Let $\left\{X_{m}\right\}_{m \geqslant 1}, X$ be $\mathcal{S}_{n}^{\prime}\left(\mathbb{R}^{d}\right)$-valued random variables. The following conditions are equivalent:

- $X_{m} \Rightarrow X$ in $\mathcal{S}_{n}^{\prime}\left(\mathbb{R}^{d}\right)$ given the strong topology,

- $X_{m} \Rightarrow X$ in $\mathcal{S}_{n}^{\prime}\left(\mathbb{R}^{d}\right)$ given the weak topology,

- $\mathcal{L}_{X_{m}}(f) \rightarrow \mathcal{L}_{X}(f)$ for all $f \in \mathcal{S}_{n}\left(\mathbb{R}^{d}\right)$.

Since both notions of convergence are equivalent, we shall just write $X_{m} \Rightarrow X$ in $\mathcal{S}_{n}^{\prime}\left(\mathbb{R}^{d}\right)$ in the sequel.

Proofs of Theorems 2.1 and 2.2: We refer to Fernique (1967) for the stated results in the more general framework in terms of nuclear spaces. For the special case $\mathcal{S}^{\prime}\left(\mathbb{R}^{d}\right) \equiv \mathcal{S}_{0}^{\prime}\left(\mathbb{R}^{d}\right)$, we refer to Biermé et al. (2017) where self-contained and simplified proofs can be found. These results can then be extended for $\mathcal{S}_{1}^{\prime}\left(\mathbb{R}^{d}\right)$ by following the idea of Proposition 2.1 from Dobrushin (1979). Let us quote that fixing a function $\psi \in \mathcal{S}\left(\mathbb{R}^{d}\right) \backslash \mathcal{S}_{1}\left(\mathbb{R}^{d}\right)$, one can define the continuous map $U: \mathcal{S}_{1}^{\prime}\left(\mathbb{R}^{d}\right) \rightarrow \mathcal{S}^{\prime}\left(\mathbb{R}^{d}\right)$ by $U(L)(f)=L(\pi(f))$, where for $f \in \mathcal{S}\left(\mathbb{R}^{d}\right)$,

$$
\pi(f)=f-c(f) \psi \in \mathcal{S}_{1}\left(\mathbb{R}^{d}\right),
$$

with $c(f)=\int_{\mathbb{R}^{d}} f(x) d x / \int_{\mathbb{R}^{d}} \psi(x) d x$. Hence any $\mathcal{S}_{1}^{\prime}\left(\mathbb{R}^{d}\right)$-valued random variable $X$ coincides with the restriction of an $\mathcal{S}^{\prime}\left(\mathbb{R}^{d}\right)$-valued random variable $Y$, defined by $Y(f)=X(\pi(f)), f \in \mathcal{S}\left(\mathbb{R}^{d}\right)$. By using the so-defined map $U$ and applying results on $\mathcal{S}^{\prime}\left(\mathbb{R}^{d}\right)$, the desired results for $\mathcal{S}_{1}^{\prime}\left(\mathbb{R}^{d}\right)$ follow.

2.2. A generalized random ball model. Now we define the random ball model on $\mathbb{R}^{d}$. Throughout, the operator-scaling is associated to a $d \times d$ real matrix $E$, of which all eigenvalues have strictly positive real parts, denoted by $a_{1} \geqslant \cdots \geqslant a_{d}>0$. Let $q=\operatorname{tr}(E)>0$ be the trace of the matrix $E$.

We consider the kernel operator defined for $(x, r) \in \mathbb{R}^{d} \times(0, \infty)$ and $f \in \mathcal{S}\left(\mathbb{R}^{d}\right)$, by

$$
T_{r}^{E} f(x):=\int_{\mathbb{R}^{d}} K_{r}^{E}(x, y) f(y) d y \quad \text { with } K_{r}^{E}(x, y):=\mathbf{1}_{B_{E}(x, r)}(y) .
$$

Here and throughout, $B_{E}(x, r)$ is the shifted and scaled "ball" given by

$$
B_{E}(x, r)=x+r^{E} B, \quad x \in \mathbb{R}^{d}, r>0,
$$

based on a fixed bounded measurable set $B \subset \mathbb{R}^{d}$ with $0 \in B, v_{B}:=\operatorname{Leb}_{d}(B) \in$ $(0, \infty)$ and $\operatorname{Leb}_{d}(\partial B)=0$, where $\operatorname{Leb}_{d}$ is the Lebesgue measure on $\mathbb{R}^{d}$. Thus 
$v_{r}:=\operatorname{Leb}_{d}\left(B_{E}(x, r)\right)=r^{q} v_{B}$. Note that we keep the name "random ball" from the original model but here the set $B$ can be a much more general set than a ball. We only assume that $B$ is a set of finite perimeter in the sense that

$$
\operatorname{Per}(B):=\sup \left\{\int_{B} \operatorname{div} \varphi(x) d x: \varphi \in \mathcal{C}_{c}^{1}\left(\mathbb{R}^{d}, \mathbb{R}^{d}\right),\|\varphi\|_{\infty} \leqslant 1\right\}<\infty,
$$

where $\mathcal{C}_{c}^{1}\left(\mathbb{R}^{d}, \mathbb{R}^{d}\right)$ is the set of continuously differentiable functions with compact support (e.g. $B$ can be any bounded convex set). According to Theorem 14 in Galerne (2011), (2.3) is equivalent to the fact that the covariogram $g_{B}: \mathbb{R}^{d} \ni x \mapsto$ $\operatorname{Leb}_{d}(B \cap(x+B))$ of the set $B$ is Lipschitz, and thus there exists $C>0$ such that

$$
\operatorname{Leb}_{d}(B \Delta(x+B))=2\left(g_{B}(0)-g_{B}(x)\right) \leqslant C|x|, \text { for all } x \in \mathbb{R}^{d} .
$$

We first define the model as a collection of random variables indexed by $f \in$ $\mathcal{S}\left(\mathbb{R}^{d}\right)$, and then prove the existence of regularizations afterwards. The rescaled random ball field is defined as

$$
X_{\rho}^{E}(f):=\int_{\mathbb{R}^{d} \times \mathbb{R}_{+} \times \mathbb{R}} m T_{r}^{E} f(x) \mathcal{N}_{\rho}(d x, d r, d m), \quad f \in \mathcal{S}\left(\mathbb{R}^{d}\right),
$$

where $\mathcal{N}_{\rho}$ is a Poisson random measure on $\mathbb{R}^{d} \times \mathbb{R}_{+} \times \mathbb{R}$ with intensity

$$
\lambda(\rho) d x F(d r / \rho) G(d m) .
$$

Intuitively, the origins of random balls are distributed as a homogeneous Poisson process with intensity $\lambda(\rho)$, and each random ball is scaled with a random radius with distribution $F_{\rho}(d r):=F(d r / \rho)$, and is associated with a random weight $m$ with distribution $G$. Positions, scalings and weights are assumed to be independent. There are a few natural assumptions on $F$ and $G$. First, the expected volume of a random ball is assumed to be finite. That is,

$$
v_{B} \int_{\mathbb{R}_{+}} r^{q} F(d r)<\infty .
$$

Moreover, we assume that, for some $C_{\beta}>0$,

$$
F(d r)=p(r) d r \quad \text { with } \quad p(r) \sim C_{\beta} r^{-1-\beta} \quad \text { as } r \rightarrow 0^{q-\beta},
$$

with the convention, $0^{\delta}=0$ if $\delta>0$ and $0^{\delta}=\infty$ if $\delta<0$. This condition is introduced in a compact form for both zoom-in/out scalings to be explained in Section 2.3. It reads as $p(r)$ is regularly varying at 0 with index $-1-\beta$, only when $\beta<q$; otherwise (2.6) will be violated. Similarly, $p(r)$ is regularly varying at infinity with index $-1-\beta$ when $\beta>q$. Next, for the random weights, their distribution $G$ is assumed to be integrable and in the domain of attraction of certain stable distribution $S_{\alpha}(\sigma, b, 0)$ with $\alpha \in(1,2], \sigma>0, b \in[-1,1]$. That is, for independent random variables $M_{i}$ with common distribution $G$,

$$
\frac{M_{1}+\cdots+M_{n}}{n^{1 / \alpha}} \Rightarrow S_{\alpha}(\sigma, b, 0) \quad \text { with } \alpha \in(1,2] .
$$

A standard reference for stable distributions and processes is Samorodnitsky and Taqqu (1994). Under (2.6) and (2.8) with $\alpha>1$, the random field (2.5) is 
well-defined and integrable. This follows from the fact

$$
\begin{aligned}
\mathbb{E}\left(\left|X_{\rho}^{E}(f)\right|\right) & \leqslant \int_{\mathbb{R}^{d} \times \mathbb{R}_{+} \times \mathbb{R}}|m| T_{r}^{E}|f|(x) \lambda(\rho) d x F(d r / \rho) G(d m) \\
& \leqslant \lambda(\rho) \rho^{q} \mathbb{E}(|M|) v_{B}\|f\|_{L^{1}} \int_{\mathbb{R}^{+}} r^{q} F(d r),
\end{aligned}
$$

where $M$ is a real random variable of distribution $G$ and $\|f\|_{L^{1}}:=\int_{\mathbb{R}^{d}}|f(y)| d y$. Hence, a centered rescaled random ball field can be defined by

$$
Y_{\rho}^{E}(f):=X_{\rho}^{E}(f)-\mathbb{E}\left(X_{\rho}^{E}(f)\right), \quad f \in \mathcal{S}\left(\mathbb{R}^{d}\right) .
$$

We come to the generalized random field interpretation of $X_{\rho}^{E}$ and $Y_{\rho}^{E}$.

Proposition 2.3. Under assumption (2.6), $X_{\rho}^{E}$ and $Y_{\rho}^{E}$ are almost surely elements of $\mathcal{S}^{\prime}\left(\mathbb{R}^{d}\right)$ and therefore of $\mathcal{S}_{1}^{\prime}\left(\mathbb{R}^{d}\right)$. As a consequence, they admit regularizations in $\mathcal{S}^{\prime}\left(\mathbb{R}^{d}\right)$ and therefore in $\mathcal{S}_{1}^{\prime}\left(\mathbb{R}^{d}\right)$.

Proof: Let us quote that $f \mapsto T_{r}^{E} f(x) \in \mathcal{S}^{\prime}\left(\mathbb{R}^{d}\right)$, and moreover for all $k \geqslant 0$,

$$
\left|T_{r}^{E} f(x)\right| \leqslant\left(\int_{B_{E}(x, r)}(1+|y|)^{-k} d y\right) \sup _{z \in \mathbb{R}^{d}}(1+|z|)^{k}|f(z)| .
$$

It follows that,

$$
\left|X_{\rho}^{E}(f)\right| \leqslant \mathcal{C}_{\rho, k}^{E} \sup _{z \in \mathbb{R}^{d}}(1+|z|)^{k}|f(z)|
$$

with

Note that

$$
\mathcal{C}_{\rho, k}^{E}:=\int_{\mathbb{R}^{d} \times \mathbb{R}_{+} \times \mathbb{R}}|m| \int_{B_{E}(x, r)}(1+|y|)^{-k} d y \mathcal{N}_{\rho}(d x, d r, d m) .
$$

$$
\begin{aligned}
\mathbb{E}\left(\mathcal{C}_{\rho, k}^{E}\right) & =\lambda(\rho) \int_{\mathbb{R}^{d} \times \mathbb{R}_{+} \times \mathbb{R}}|m| \int_{B_{E}(x, r)}(1+|y|)^{-k} d y d x F_{\rho}(d r) G(d m) \\
& =\lambda(\rho) \rho^{q} \mathbb{E}(|M|) v_{B} \int_{\mathbb{R}^{+}} r^{q} F(d r)\left(\int_{\mathbb{R}^{d}}(1+|y|)^{-k} d y\right),
\end{aligned}
$$

which is finite under assumption (2.6) as soon as $k>d$. Hence, $\mathcal{C}_{\rho, k}^{E}<\infty$ a.s. for $k>d$, so that $X_{\rho}^{E} \in \mathcal{S}^{\prime}\left(\mathbb{R}^{d}\right)$ a.s. Since we also have $f \mapsto \mathbb{E}\left(X_{\rho}^{E}(f)\right) \in \mathcal{S}^{\prime}\left(\mathbb{R}^{d}\right)$ by taking expectation in the previous computations, it follows that the centered field $Y_{\rho}^{E}$ is also in $\mathcal{S}^{\prime}\left(\mathbb{R}^{d}\right)$ a.s. The last part of the proposition is easy since to obtain a regularization in $\mathcal{S}^{\prime}\left(\mathbb{R}^{d}\right)$ of a process $X$ which is almost surely element of $\mathcal{S}^{\prime}\left(\mathbb{R}^{d}\right)$, it suffices to modify it by setting $X(\omega) \equiv 0$ for the $\omega \in \Omega$ such that $X(\omega) \notin \mathcal{S}^{\prime}\left(\mathbb{R}^{d}\right)$, see Fernique (1967), p.40.

The limit theorems will be based on the characteristic functionals of the centered rescaled random fields

$$
\mathcal{L}_{Y_{\rho}^{E}}(f)=\mathbb{E} \exp \left(i Y_{\rho}^{E}(f)\right)=\exp \left(\int_{\mathbb{R}^{d} \times \mathbb{R}_{+}} \phi_{G}\left(T_{r}^{E} f(x)\right) \lambda(\rho) d x F_{\rho}(d r)\right), f \in \mathcal{S}_{n}\left(\mathbb{R}^{d}\right),
$$

with

$$
\phi_{G}(t):=\int\left(e^{i m t}-1-i m t\right) G(d m)=\mathcal{L}_{M}(t)-1-i t \mathbb{E}(M), \quad t \in \mathbb{R},
$$

where $M$ is a real random variable of distribution $G$ satisfying (2.8). 
2.3. Zoom-in/out scalings and four regimes. There are two scalings to be considered in the limit theorems. Recall $F_{\rho}(d r)=F(d r / \rho)$. The case $\rho \rightarrow \infty$ corresponds to enlarging the size of each ball, and $\rho \rightarrow 0$ corresponds to shrinking the size of each ball. We refer to the two scalings as the zoom-in and zoom-out scalings, respectively.

Next, for each type of scaling, there are four qualitatively different regimes. Since the spatial dependence of the random field is essentially determined by overlaps of random balls, heuristically we compute the expected weight of rescaled balls covering a fixed point $y$, denoted by $m(\rho)$, independent from $y$ by stationarity. It is natural to expect $m(\rho) \rightarrow c \in[0, \infty]$, and we distinguish $\infty,(0, \infty)$ and 0 as three different cases. Take the zoom-in scaling case first. Clearly only small balls, say with radius less than 1 (before the $\rho$-scaling and the constant 1 is irrelevant) should matter, and we compute

$$
\begin{aligned}
m_{\mathrm{in}}(\rho) & :=\mathbb{E}\left(\int_{\mathbb{R}^{d} \times \mathbb{R}_{+} \times \mathbb{R}} m \mathbf{1}_{\left\{y \in B_{E}(x, r)\right\}} \mathbf{1}_{\{r \leqslant 1\}} \mathcal{N}_{\rho}(d x, d r, d m)\right) \\
& =\mathbb{E}(M) \lambda(\rho) v_{B} \int_{0}^{1} r^{q} F_{\rho}(d r),
\end{aligned}
$$

with

$$
\lambda(\rho) \int_{0}^{1} r^{q} F_{\rho}(d r) \sim\left(C_{\beta} \int_{0}^{1} r^{q-\beta-1} d r\right) \lambda(\rho) \rho^{\beta} \quad \text { as } \rho \rightarrow \infty .
$$

Similarly for the zoom-out case, we compute for number of balls with radius larger than 1 ,

$$
\begin{aligned}
m_{\text {out }}(\rho) & :=\mathbb{E}\left(\int_{\mathbb{R}^{d} \times \mathbb{R}_{+} \times \mathbb{R}} m \mathbf{1}_{\left\{y \in B_{E}(x, r)\right\}} \mathbf{1}_{\{r>1\}} \mathcal{N}_{\rho}(d x, d r, d m)\right) \\
& =\mathbb{E}(M) \lambda(\rho) v_{B} \int_{1}^{\infty} r^{q} F_{\rho}(d r),
\end{aligned}
$$

with

$$
\lambda(\rho) \int_{1}^{\infty} r^{q} F_{\rho}(d r) \sim\left(C_{\beta} \int_{1}^{\infty} r^{q-\beta-1} d r\right) \lambda(\rho) \rho^{\beta} \quad \text { as } \rho \rightarrow 0 .
$$

The calculations above made use of (2.7), and also explain why it is a reasonable assumption. Notice that the constant is qualitatively irrelevant, only the common term $\lambda(\rho) \rho^{\beta}$ matters, and both cases of scaling can be summarized in the compact form of $\rho \rightarrow 0^{\beta-q}$.

In summary, there are naturally three regimes of interest, characterized by

$$
\lambda(\rho) \rho^{\beta} \rightarrow \begin{cases}\infty & (\text { dense regime), } \\ c \in(0, \infty) & \left(\text { intermediate regime), as } \rho \rightarrow 0^{\beta-q},\right. \\ 0 & (\text { (very-)sparse regime), }\end{cases}
$$

where within the case $\lambda(\rho) \rho^{\beta} \rightarrow 0$ we shall further identify two sub-regimes, named as sparse and very-sparse regimes in the sequel. We shall establish limit theorems for different regimes separately, and in each regime our limit theorem and the proof unify both zoom-in and zoom-out scalings (only zoom-out scaling in the very-sparse regime). Furthermore, in each regime we specify two parameters, $\beta$ on the tails of the radius of random balls, and $n$ indicating the zoom-in $(n=1)$ and zoom-out $(n=0)$ scalings. 


\section{Scaling limits}

We will treat the four regimes separately. In each regime, we first introduce the limit field as stochastic integral, then show the existence of its generalized random field version by Minlos-Bochner's theorem and then prove the weak convergence by Lévy's continuity theorem. For easy reading, all the proofs of this section are postponed to Section 6. The limit fields appearing here are further investigated in the next sections.

3.1. Dense regime. In the dense regime, we consider

$$
\lambda(\rho) \rho^{\beta} \rightarrow \infty \text { as } \rho \rightarrow 0^{\beta-q},
$$

and the admissible range of parameters $\beta$ and $n$ is

$$
\begin{array}{lll}
\beta \in(q, \alpha q) & n=0 & \text { zoom-out scaling, } \\
\beta \in\left(q-a_{d}, q\right) & n=1 & \text { zoom-in scaling. }
\end{array}
$$

The following field appears in the limit. Let $\alpha \in(1,2], \sigma>0$ and $b \in[-1,1]$ be given by (2.8) and $C_{\beta}>0$ be given by (2.7). Let $M_{\alpha, \beta}$ be an $\alpha$-stable random measure on $\mathbb{R}^{d} \times \mathbb{R}_{+}$with control measure $\sigma^{\alpha} C_{\beta} r^{-1-\beta} d r d x$, and constant skewness function $b$. For $f \in \mathcal{S}_{n}\left(\mathbb{R}^{d}\right)$, let us define the stochastic integral

$$
Z_{\alpha, \beta}^{E}(f):=\int_{\mathbb{R}^{d} \times \mathbb{R}_{+}} T_{r}^{E} f(x) M_{\alpha, \beta}(d r, d x) .
$$

See Samorodnitsky and Taqqu (1994) for more background on stochastic integrals with respect to $\alpha$-stable random measures.

Proposition 3.1. Let $\alpha \in(1,2]$. For $\beta, n$ as in (3.1), the process $Z_{\alpha, \beta}^{E}:=$ $\left\{Z_{\alpha, \beta}^{E}(f)\right\}_{f \in \mathcal{S}_{n}\left(\mathbb{R}^{d}\right)}$ in (3.2) is well-defined, has characteristic functional

$\mathcal{L}_{Z_{\alpha, \beta}^{E}}(f)=\exp \left\{-C_{\beta} \sigma^{\alpha} \int_{\mathbb{R}^{d} \times \mathbb{R}_{+}}\left|T_{r}^{E} f(x)\right|^{\alpha}\left(1-i b \epsilon\left(T_{r}^{E} f(x)\right) \tan \frac{\alpha \pi}{2}\right) r^{-1-\beta} d r d x\right\}$,

where $\epsilon(s)=\operatorname{sign}(s)$, and admits a version with values in $\mathcal{S}_{n}^{\prime}\left(\mathbb{R}^{d}\right)$.

Then, we can consider weak convergence in $\mathcal{S}_{n}^{\prime}\left(\mathbb{R}^{d}\right)$ and state the limit theorem in the dense regime.

Theorem 3.2. Suppose that the assumptions (2.7) and (2.8) on $F$ and $G$ hold. Under (3.1), if $n_{1}(\rho):=\rho^{\beta} \lambda(\rho) \rightarrow \infty$ as $\rho \rightarrow 0^{\beta-q}$, then

$$
\frac{1}{n_{1}(\rho)^{1 / \alpha}} Y_{\rho}^{E} \Rightarrow Z_{\alpha, \beta}^{E} \quad \text { as } \rho \rightarrow 0^{\beta-q}
$$

in $\mathcal{S}_{n}^{\prime}\left(\mathbb{R}^{d}\right)$.

Remark 3.3. We let $\left\{Z_{\alpha, \beta}^{E}(f)\right\}_{f \in \mathcal{S}_{n}\left(\mathbb{R}^{d}\right)}$ denote the stochastic process indexed by $f$ via (3.2), and the same notation $Z_{\alpha, \beta}^{E}$ in Theorem 3.2 for the corresponding version taking values in $\mathcal{S}_{n}^{\prime}\left(\mathbb{R}^{d}\right)$. Similar notations are used for the other regimes. 
3.2. Intermediate regime. In the intermediate regime, we consider

$$
\lambda(\rho) \rho^{\beta} \rightarrow a^{q-\beta} \text { as } \rho \rightarrow 0^{\beta-q} \quad \text { with } \quad a \in(0, \infty)
$$

The admissible range of parameters $\beta$ and $n$ is the same (3.1) as in the dense regime. In this case, the limit field is represented by a Poisson integral. For $a \in(0, \infty)$ and $f \in \mathcal{S}\left(\mathbb{R}^{d}\right)$, we first define

$$
T_{r, a}^{E} f(x):=\int_{\mathbb{R}^{d}} \mathbf{1}_{a^{-E} B_{E}(x, r)}(y) f(y) d y=T_{r / a}^{E} f\left(a^{-E} x\right)
$$

and we consider the Poisson integral $J_{a, \alpha, \beta}^{E}$ defined, for $f \in \mathcal{S}_{n}\left(\mathbb{R}^{d}\right)$, by

$$
J_{a, \alpha, \beta}^{E}(f):=\int_{\mathbb{R}^{d} \times \mathbb{R}_{+} \times \mathbb{R}_{+}} m T_{r, a}^{E} f(x) \tilde{\mathcal{N}}_{\beta}(d r, d x, d m),
$$

where $\tilde{\mathcal{N}}_{\beta}$ is the compensated Poisson random measure on $\mathbb{R}^{d} \times \mathbb{R}_{+} \times \mathbb{R}_{+}$with intensity $C_{\beta} r^{-1-\beta} d x d r G(d m)$, with $C_{\beta}>0$ given in (2.7). For more background on Poisson integrals, see for example Kallenberg (1997).

Proposition 3.4. Let $a \in(0, \infty)$. For $\beta, n$ as in (3.1), the process $J_{a, \alpha, \beta}^{E}$ in (3.6) is well-defined on $\mathcal{S}_{n}\left(\mathbb{R}^{d}\right)$, has characteristic functional

$$
\mathcal{L}_{J_{a, \alpha, \beta}^{E}}(f)=\exp \left\{\int_{\mathbb{R}^{d} \times \mathbb{R}_{+}} \phi_{G}\left(T_{r, a}^{E} f(x)\right) C_{\beta} r^{-1-\beta} d r d x\right\},
$$

where $\phi_{G}$ is defined by (2.10) and admits a version with values in $\mathcal{S}_{n}^{\prime}\left(\mathbb{R}^{d}\right)$.

The limit theorem in the intermediate regime is the following.

Theorem 3.5. Suppose that the assumptions (2.7) and (2.8) on F and $G$ hold. Under (3.1) and (3.4),

$$
Y_{\rho}^{E} \Rightarrow J_{a, \alpha, \beta}^{E} \quad \text { as } \rho \rightarrow 0^{\beta-q}
$$

in $\mathcal{S}_{n}^{\prime}\left(\mathbb{R}^{d}\right)$.

3.3. Sparse regime. The sparse regime correspond to

$$
\lambda(\rho) \rho^{\beta} \rightarrow 0 \text { as } \rho \rightarrow 0^{\beta-q} \quad \text { with } \quad \lambda(\rho) \rightarrow 0^{q-\beta} .
$$

The admissible range of parameters of $\beta$ and $n$ is

$$
\begin{array}{lll}
\beta \in(q, \alpha q) & n=0 & \text { zoom-out scaling, } \\
\beta \in\left(q^{2} /\left(q+a_{d}\right), q\right) & n=1 & \text { zoom-in scaling. }
\end{array}
$$

Set $\gamma=\beta / q \in\left(q /\left(q+a_{d}\right), 1\right) \cup(1, \alpha)$. Let $M_{\gamma}^{(1)}$ be a $\gamma$-stable random measure having control measure $\sigma_{1, \gamma} d x$ with

$$
\sigma_{1, \gamma}:=v_{B}\left(C_{\beta} q^{-1} \int_{\mathbb{R}_{+}}(1-\cos (r)) r^{-1-\gamma} d r \int_{\mathbb{R}}|m|^{\gamma} G(d m)\right)^{1 / \gamma},
$$

and constant skewness function

$$
b_{\gamma}:=-\frac{\int_{\mathbb{R}} \epsilon(m)|m|^{\gamma} G(d m)}{\int_{\mathbb{R}}|m|^{\gamma} G(d m)} .
$$

We define, for $f \in \mathcal{S}\left(\mathbb{R}^{d}\right)$,

$$
Z_{\gamma}^{(1)}(f):=\int_{\mathbb{R}^{d}} f(x) M_{\gamma}^{(1)}(d x)
$$


Note that $Z_{\gamma}^{(1)}(f)$ is well-defined since $f \in \mathcal{S}\left(\mathbb{R}^{d}\right) \subset L^{\gamma}\left(\mathbb{R}^{d}\right)$ and its characteristic functional is given by

$$
\mathcal{L}_{Z_{\gamma}^{(1)}}(f)=\exp \left(-\sigma_{1, \gamma}^{\gamma} \int_{\mathbb{R}^{d}}|f(x)|^{\gamma}\left(1-i b_{\gamma} \epsilon(f(x)) \tan \frac{\gamma \pi}{2}\right) d x\right) .
$$

Proposition 3.6. For $\alpha \in(1,2]$ and $\gamma \in\left(q /\left(q+a_{d}\right), 1\right) \cup(1, \alpha)$, the process $Z_{\gamma}^{(1)}$ admits a version with values in $\mathcal{S}_{0}^{\prime}\left(\mathbb{R}^{d}\right) \subset \mathcal{S}_{1}^{\prime}\left(\mathbb{R}^{d}\right)$.

Theorem 3.7. Suppose that the assumptions (2.7) and (2.8) on $F$ and $G$ hold. Under (3.8) and (3.9) with $n_{2}(\rho):=\left(\lambda(\rho)^{1 / \beta} \rho\right)^{q}$ and $\gamma=\beta / q$, we have

$$
\frac{1}{n_{2}(\rho)} Y_{\rho}^{E} \Rightarrow Z_{\gamma}^{(1)} \quad \text { as } \rho \rightarrow 0^{\beta-q}
$$

in $\mathcal{S}_{n}^{\prime}\left(\mathbb{R}^{d}\right)$.

Remark 3.8. Note that the result in the case $\beta \in\left(q^{2} /\left(q+a_{d}\right), q\right)$ is also new for the isotropic case when $E=I_{d}$ (the identity matrix).

3.4. Very-sparse regime. In this regime, consider

$$
\lambda(\rho) \rho^{\beta} \rightarrow 0, \lambda(\rho) \rightarrow \infty \text { as } \rho \rightarrow 0 .
$$

The admissible range of parameters for the very-sparse regime is

$$
\beta \in(\alpha q, \infty) \quad n=0 \quad \text { zoom-out scaling. }
$$

Let $M_{\alpha}^{(2)}$ be a $\alpha$-stable random measure having control measure $\sigma_{2, \alpha} d x$ with

$$
\sigma_{2, \alpha}:=\sigma v_{B}\left(\int_{\mathbb{R}_{+}} r^{\alpha q} F(d r)\right)^{1 / \alpha}
$$

and constant skewness function $b$. For $f \in \mathcal{S}\left(\mathbb{R}^{d}\right)$, we set

$$
Z_{\alpha}^{(2)}(f):=\int_{\mathbb{R}^{d}} f(x) M_{\alpha}^{(2)}(d x) .
$$

Proposition 3.9. For $\alpha \in(1,2]$, the process $Z_{\alpha}^{(2)}$ admits a version with values in $\mathcal{S}_{0}^{\prime}\left(\mathbb{R}^{d}\right)$.

Theorem 3.10. Suppose that the assumptions (2.7) and (2.8) on $F$ and $G$ hold. Under (3.11) and (3.12), with $n_{3}(\rho):=\lambda(\rho)^{1 / \alpha} \rho^{q}$,

$$
\frac{1}{n_{3}(\rho)} Y_{\rho}^{E} \Rightarrow Z_{\alpha}^{(2)} \quad \text { as } \rho \rightarrow 0
$$

in $\mathcal{S}_{0}^{\prime}\left(\mathbb{R}^{d}\right)$.

3.5. Summary. For comparison, we summarize in a single statement the limit theorems of the different regimes. 
Theorem 3.11. Suppose that the assumptions (2.7) and (2.8) on $F$ and $G$ hold. We have the following weak convergence in $\mathcal{S}_{n}^{\prime}\left(\mathbb{R}^{d}\right)$ :

$$
\begin{aligned}
& \text { (dense) } \frac{1}{\left(\rho^{\beta} \lambda(\rho)\right)^{1 / \alpha}} Y_{\rho}^{E} \Rightarrow Z_{\alpha, \beta}^{E} \quad \text { if } \lambda(\rho) \rho^{\beta} \rightarrow \infty, \beta, n \text { as in }(3.1), \\
& \text { (intermediate) } Y_{\rho}^{E} \Rightarrow J_{a, \alpha, \beta}^{E} \quad \text { if } \lambda(\rho) \rho^{\beta} \rightarrow a^{q-\beta} \in(0, \infty), \beta, n \text { as in }(3.1), \\
& \text { (sparse) } \frac{1}{\left(\rho^{\beta} \lambda(\rho)\right)^{q / \beta}} Y_{\rho}^{E} \Rightarrow Z_{\beta / q}^{(1)} \quad \text { if } \lambda(\rho) \rho^{\beta} \rightarrow 0, \lambda(\rho) \rightarrow 0^{q-\beta}, \beta, n \text { as in (3.9), } \\
& \text { (very sparse) } \frac{1}{\rho^{q} \lambda(\rho)^{1 / \alpha}} Y_{\rho}^{E} \Rightarrow Z_{\alpha}^{(2)} \text { if } \lambda(\rho) \rho^{\beta} \rightarrow 0, \lambda(\rho) \rightarrow \infty, \beta, n \text { as in }(3.12),
\end{aligned}
$$

where in all cases the limit is considered as $\rho \rightarrow 0^{\beta-q}$.

\section{Properties of the limit fields}

In this section, we provide some properties of the limit generalized random fields. In the dense and intermediate regimes, the limit generalized random fields explicitly depend on $E$, and in particular so are their anisotropic properties. For the sparse and very-sparse regimes, all the dependence structures in the discrete models are not observable in the limit, and thus the limit generalized random fields have no specific anisotropic properties. Following Dobrushin (1979), using duality, we can define the following groups of transformations on $\mathcal{S}_{n}\left(\mathbb{R}^{d}\right)$ :

- the group of shift transformations $\mathcal{T}=\left\{\tau_{h}\right\}_{h \in \mathbb{R}^{d}}$ :

$$
\tau_{h} f(t)=f(t-h), \quad f \in \mathcal{S}_{n}\left(\mathbb{R}^{d}\right), h \in \mathbb{R}^{d}, t \in \mathbb{R}^{d} ;
$$

- the group of $E$-operator-scaling transformations $\Delta^{E}=\left\{\delta_{c}^{E}\right\}_{c \in(0, \infty)}$ :

$$
\delta_{c}^{E} f(t)=c^{-q} f\left(c^{-E} t\right), \quad f \in \mathcal{S}_{n}\left(\mathbb{R}^{d}\right), c \in(0, \infty), q=\operatorname{tr}(E), t \in \mathbb{R}^{d} .
$$

Their analogous $\mathcal{T}, \Delta^{E}$ on $\mathcal{S}_{n}^{\prime}\left(\mathbb{R}^{d}\right)$ are then defined by

$$
\tau_{h} L(f):=L\left(\tau_{h} f\right) \text {, and } \delta_{c}^{E} L(f):=L\left(\delta_{c}^{E} f\right),
$$

for $L \in \mathcal{S}_{n}^{\prime}\left(\mathbb{R}^{d}\right)$. Let us note that when the tempered distribution $L$ is given by a function $g$, one recovers that $\tau_{h} L$ is given by the function $g(\cdot+h)$ and $\delta_{c}^{E} L$ is given by the function $g\left(c^{E} \cdot\right)$, thanks to the normalization term.

Proposition 4.1. Let $\alpha \in(1,2]$. For $\beta, n$ as in (3.1), the generalized random field $Z_{\alpha, \beta}^{E}$ in (3.2) is

- shift-invariant: $\forall h \in \mathbb{R}^{d}$,

$$
\tau_{h} Z_{\alpha, \beta}^{E} \stackrel{d}{=} Z_{\alpha, \beta}^{E},
$$

- $(E, H)$-operator-scaling for $H=\frac{q-\beta}{\alpha} \in(-q(1-1 / \alpha), 0) \cup\left(0, a_{d} / \alpha\right): \forall c>0$,

$$
\delta_{c}^{E} Z_{\alpha, \beta}^{E} \stackrel{d}{=} c^{H} Z_{\alpha, \beta}^{E} .
$$

Let us remark that, following Dobrushin (1979), the first property is called the stationary $n$-th increments while the second one with $E=I_{d}$ is the self-similarity property. 
Proof: It suffices to compute the characteristic functional. Observe that for $f \in$ $\mathcal{S}_{n}(\mathbb{R})$, one has for all $h \in \mathbb{R}^{d}$,

$$
Z_{\alpha, \beta}^{E}\left(\tau_{h} f\right)=\int_{\mathbb{R}^{d} \times \mathbb{R}_{+}} T_{r}^{E} f(x-h) M_{\alpha, \beta}(d x, d r) \stackrel{\mathrm{d}}{=} Z_{\alpha, \beta}^{E}(f),
$$

by a change of variable, while for all $c>0$,

$$
\begin{aligned}
Z_{\alpha, \beta}^{E}\left(\delta_{c}^{E} f\right) & =\int_{\mathbb{R}^{d} \times \mathbb{R}_{+}} T_{r}^{E} \delta_{c}^{E} f(x) M_{\alpha, \beta}(d x, d r) \\
& =\int_{\mathbb{R}^{d} \times \mathbb{R}_{r / c}} T_{r}^{E} f\left(c^{-E} x\right) M_{\alpha, \beta}(d x, d r) \\
& \stackrel{\mathrm{d}}{=} c^{(q-\beta) / \alpha} \int_{\mathbb{R}^{d} \times \mathbb{R}} T_{r}^{E} f(x) M_{\alpha, \beta}(d x, d r)=c^{(q-\beta) / \alpha} Z_{\alpha, \beta}^{E}(f),
\end{aligned}
$$

where the third step also followed from a change of variable argument.

For the intermediate case, the limit random field $J_{a, \alpha, \beta}^{E}$ in (3.6) is not $E$-operatorscaling but it has aggregate $E$-operator-scaling property as described below, generalizing aggregate similarity property introduced by Biermé et al. (2010).

Proposition 4.2. Under the assumption of Theorem 3.5,

$$
\delta_{k^{1 /(q-\beta)}}^{E} J_{a, \alpha, \beta}^{E} \stackrel{\mathrm{d}}{=} \sum_{i=1}^{k} J_{a, \alpha, \beta}^{E,(i)}, \text { for all } k \in \mathbb{N},
$$

where $\left\{J_{a, \alpha, \beta}^{E,(i)}\right\}_{i=1, \ldots, k}$ are i.i.d. copies of $J_{a, \alpha, \beta}^{E}$. Furthermore,

$$
\frac{1}{a^{(q-\beta) / \alpha}} J_{a, \alpha, \beta}^{E} \Rightarrow Z_{\alpha, \beta}^{E} \quad \text { as } a \rightarrow 0^{\beta-q} .
$$

Proof: The first part of the proof follows from straightforward calculation of characteristic functionals, with a similar change of variable argument as above. The second part of the proof follows from convergence of characteristic functionals for random variables in the domain of attractions of $S_{\alpha}(\sigma, b, 0)$. The details are omitted.

At last, remark that in the sparse and very-sparse regimes, the limit random fields have essentially no dependence structure, as the limit random fields are stochastic integrals with respect to stable random measures with constant control measure on $\mathbb{R}^{d}$. Thus they inherit no specific anisotropic properties. Nevertheless, for any $E^{\prime}$ satisfying the same assumption as $E$ with possibly different eigenvalues, writing $q^{\prime}=\operatorname{tr}\left(E^{\prime}\right)$, it can be shown that

$$
\delta_{c}^{E^{\prime}} Z_{\theta}^{(i)} \stackrel{\mathrm{d}}{=} c^{\frac{1-\theta}{\theta} q^{\prime}} Z_{\theta}^{(i)}
$$

for $i=1,2$ with legitimate parameter $\theta$.

\section{Comments on pointwise representation}

Given a tempered distribution $L \in \mathcal{S}^{\prime}\left(\mathbb{R}^{d}\right)$, it is a natural question to wonder if it may be represented by a Borel measurable function $g$, that is

$$
\forall f \in \mathcal{S}\left(\mathbb{R}^{d}\right), \quad L(f)=\int_{\mathbb{R}^{d}} f(t) g(t) d t .
$$


We say that a generalized random field $X$ admits a pointwise reprensentation if there exists a measurable random field $\{\hat{X}(t)\}_{t \in \mathbb{R}^{d}}$, meaning as in Definition 9.4.1 of Samorodnitsky and Taqqu (1994) that $\hat{X}: \Omega \times \mathbb{R}^{d} \rightarrow \mathbb{R}$ is a jointly measurable function, such that

$$
X(f)=\int_{\mathbb{R}^{d}} \hat{X}(t) f(t) d t, \quad f \in \mathcal{S}\left(\mathbb{R}^{d}\right) .
$$

Conversely, we have the following property.

Proposition 5.1. Let $\{\widehat{X}(t)\}_{t \in \mathbb{R}^{d}}$ be a measurable random field. If there exists $k \in \mathbb{N}$ such that

$$
\int_{\mathbb{R}^{d}}(1+|t|)^{-k} \mathbb{E}(|\hat{X}(t)|) d t<\infty
$$

then the random field $X$, defined on $\mathcal{S}_{n}\left(\mathbb{R}^{d}\right)$ by $X(f)=\int_{\mathbb{R}^{d}} \hat{X}(t) f(t) d t$, admits a regularization that is a generalized random field. Moreover, if $\hat{X}$ is $(E, H)$-operatorscaling for some $H>0$ in the sense of (1.1), then $X$ is $(E, H)$-operator-scaling in the sense of Proposition 4.1.

Proof: Under the assumption, one checks that for all $f \in \mathcal{S}_{n}\left(\mathbb{R}^{d}\right)$,

$$
\int_{\mathbb{R}^{d}}|\hat{X}(t) f(t)| d t \leqslant \mathcal{C}_{k} \sup _{z \in \mathbb{R}^{d}}(1+|z|)^{k}|f(z)|
$$

where the random constant $\mathcal{C}_{k}=\int_{\mathbb{R}^{d}}(1+|t|)^{-k}|\hat{X}(t)| d t$ is a.s. finite. This implies that the linear random field $X$ is well-defined and a.s. continuous. Hence there exists a regularization of $X$ on $\mathcal{S}_{n}^{\prime}\left(\mathbb{R}^{d}\right)$, see Fernique (1967), p.40. The last property of the proposition is straightforward.

Our centered rescaled random ball field $Y_{\rho}^{E}$ defined in Section 2 clearly admits a pointwise representation where $\hat{Y}_{\rho}^{E}=\hat{X}_{\rho}^{E}-\mathbb{E} \hat{X}_{\rho}^{E}$ and

$$
\hat{X}_{\rho}^{E}(t)=\int_{\mathbb{R}^{d} \times \mathbb{R}_{+} \times \mathbb{R}} m K_{r}^{E}(x, t) \mathcal{N}_{\rho}(d x, d r, d m), \quad t \in \mathbb{R}^{d},
$$

with the same Poisson random measure $\mathcal{N}_{\rho}$ than in (2.5). Let us consider the limit generalized random field $Z_{\alpha, \beta}^{E}$ of the dense regime in the case of symmetric weights $(b=0)$. Actually, there are two situations that we treated separately in the following sub-sections.

5.1. The case $\beta \in\left(q-a_{d}, q\right)$ and $H=\frac{q-\beta}{\alpha} \in\left(0, a_{d} / \alpha\right)$. In this case, as proved in Proposition 5.2 below, $Z_{\alpha, \beta}^{E}$ admits a pointwise representation with

$$
\hat{Z}_{\alpha, \beta}^{E}(t)=\int_{\mathbb{R}^{d} \times \mathbb{R}_{+}}\left(\mathbf{1}_{B_{E}(x, r)}(t)-\mathbf{1}_{B_{E}(x, r)}(0)\right) M_{\alpha, \beta}(d r, d x), \quad t \in \mathbb{R}^{d},
$$

satisfying (1.1) and $M_{\alpha, \beta}$ is the same as in the representation of $Z_{\alpha, \beta}^{E}$. Let us introduce $\mathcal{C}_{E}(t)=\left\{(x, r) ; r^{-E}(x-t) \in B\right\}$ and note that

$$
\hat{Z}_{\alpha, \beta}^{E}(t)=M_{\alpha, \beta}\left(\mathcal{C}_{E}(t) \cap \mathcal{C}_{E}(0)^{c}\right)-M_{\alpha, \beta}\left(\mathcal{C}_{E}(t)^{c} \cap \mathcal{C}_{E}(0)\right), \quad t \in \mathbb{R}^{d} .
$$

Until here we do not need to assume that $M_{\alpha, \beta}$ has skewness function $b=0$.

With the assumption that $M_{\alpha, \beta}$ is symmetric, one can check that

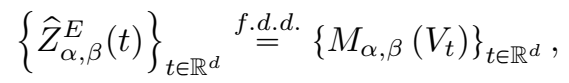


with $V_{t}=\mathcal{C}_{E}(t) \Delta \mathcal{C}_{E}(0)$. That is, the random field $\widehat{Z}_{\alpha, \beta}^{E}$ has a Chentsov's type representation (see Samorodnitsky and Taqqu, 1994, Chap. 8). In particular, for $H=\frac{q-\beta}{\alpha} \in\left(0, a_{d} / \alpha\right)$ the random field $\widehat{Z}_{\alpha, \beta}^{E}$ generalizes isotropic self-similar $(\alpha, H)$ Takenaka random fields (see p.405 of Samorodnitsky and Taqqu, 1994), defined by choosing the Euclidean unit ball for $B$ and $E=I_{d}$, with $a_{d}=1$.

The representation (5.1) allows us to provide several simulations of our operatorscaling random ball model with symmetric $\alpha$-stable $(S \alpha S)$ weights, following similar ideas as in Biermé et al. (2013). See Figures A.1-A.3 in the appendix.

Proposition 5.2. For $\beta \in\left(q-a_{d}, q\right)$, there exists a measurable version of $\hat{Z}_{\alpha, \beta}^{E}$, also denoted by $\hat{Z}_{\alpha, \beta}^{E}$, such that $Z_{\alpha, \beta}^{E}$ coincides in $\mathcal{S}_{1}^{\prime}\left(\mathbb{R}^{d}\right)$ with the generalized random field

$$
f \in \mathcal{S}\left(\mathbb{R}^{d}\right) \mapsto \int_{\mathbb{R}^{d}} \widehat{Z}_{\alpha, \beta}^{E}(t) f(t) d t .
$$

Proof: First note that

$$
\int_{\mathbb{R}^{d}}\left|\mathbf{1}_{B_{E}(x, r)}(t)-\mathbf{1}_{B_{E}(x, r)}(0)\right|^{\alpha} d x=r^{q} h\left(r^{-E} t\right)
$$

with $h(z)=\operatorname{Leb}_{d}(B \Delta(z+B))$. According to (2.4), $h$ satisfies $h(z) \leqslant C(|z| \wedge 1)$ for some constant $C>0$. It follows that

$$
\begin{aligned}
\int_{\mathbb{R}^{d} \times \mathbb{R}^{+}} \mid & \left|\mathbf{1}_{B_{E}(x, r)}(t)-\mathbf{1}_{B_{E}(x, r)}(0)\right|^{\alpha} \sigma^{\alpha} C_{\beta} r^{-1-\beta} d r d x \\
& \leqslant C \sigma^{\alpha} C_{\beta} \int_{\mathbb{R}^{+}} r^{q}\left(\left|r^{-E} t\right| \wedge 1\right) r^{-1-\beta} d r \\
& \leqslant C \sigma^{\alpha} C_{\beta} \int_{\mathbb{R}_{+}} r^{q}\left(\left\|r^{-E}\right\| \wedge 1\right) r^{-1-\beta} d r(1+|t|) \\
& =C_{\alpha, \beta}^{E}(1+|t|),
\end{aligned}
$$

with $C_{\alpha, \beta}^{E}=C \sigma^{\alpha} C_{\beta} \int_{\mathbb{R}^{+}}\left(\left\|r^{-E}\right\| \wedge 1\right) r^{q-\beta-1} d r<\infty$ and $\|\cdot\|$ the subordinated norm, since $\beta \in\left(q-a_{d}, q\right)$. Hence $\widehat{Z}_{\alpha, \beta}^{E}(t)$ is well-defined and is a $S \alpha S$ random variable with scale parameter bounded by $\left(C_{\alpha, \beta}^{E}(1+|t|)\right)^{1 / \alpha}$, for every $t \in \mathbb{R}^{d}$. According to Theorem 11.1.1 in Samorodnitsky and Taqqu (1994) there exists a measurable version of $\widehat{Z}_{\alpha, \beta}^{E}$ since

(1) $(t, x, r) \in \mathbb{R}^{d} \times \mathbb{R}^{d} \times \mathbb{R}^{+} \mapsto\left(\mathbf{1}_{B_{E}(x, r)}(t)-\mathbf{1}_{B_{E}(x, r)}(0)\right) \in \mathbb{R}$ is measurable;

(2) the control measure $\sigma^{\alpha} C_{\beta} r^{-1-\beta} d r d x$ is $\sigma$-finite.

Noting that by Property 1.2.17 in Samorodnitsky and Taqqu (1994), we have

$$
\mathbb{E}\left(\left|\hat{Z}_{\alpha, \beta}^{E}(t)\right|\right) \leqslant \mathbb{E}\left(\left|S_{\alpha}\right|\right)\left(C_{\alpha, \beta}^{E}(1+|t|)\right)^{1 / \alpha},
$$

with $S_{\alpha}$ a $S \alpha S$ random variable of scale parameter 1 , we may define $f \in \mathcal{S}\left(\mathbb{R}^{d}\right) \mapsto$ $\int_{\mathbb{R}^{d}} \hat{Z}_{\alpha, \beta}^{E}(t) f(t) d t$ that is a.s. in $\mathcal{S}^{\prime}\left(\mathbb{R}^{d}\right)$, thanks to Proposition 5.1.

Now it remains to show that the right-hand side of (5.2) has the same stable law as $Z_{\alpha, \beta}^{E}(f)=\int_{\mathbb{R}^{d} \times \mathbb{R}_{+}} T_{r}^{E} f(x) M_{\alpha, \beta}(d x, d r)$. For this we recall that

$$
\int \widehat{Z}_{\alpha, \beta}(t) f(t) d t \stackrel{\mathrm{d}}{=} \int_{\mathbb{R}^{d} \times \mathbb{R}_{+}}\left(\int_{\mathbb{R}^{d}}\left(\mathbf{1}_{B_{E}(x, r)}(t)-\mathbf{1}_{B_{E}(x, r)}(0)\right) f(t) d t\right) M_{\alpha, \beta}(d x, d r),
$$


provided that

$$
\int_{\mathbb{R}^{d}}\left|\widehat{Z}_{\alpha, \beta}^{E}(t)\right| f(t) d t<\infty \text { a.s. }
$$

see Theorem 11.4.1 in Samorodnitsky and Taqqu (1994). Since $f$ decays rapidly, the above follows from (5.3) and hence (5.4) holds. To complete the proof, it remains to remark that for $f \in \mathcal{S}_{1}\left(\mathbb{R}^{d}\right)$, one has

$$
\int_{\mathbb{R}^{d}}\left(\mathbf{1}_{B_{E}(x, r)}(t)-\mathbf{1}_{B_{E}(x, r)}(0)\right) f(t) d t=T_{r}^{E} f(x) .
$$

5.2. The case $\beta \in(q, \alpha q)$ and $H=\frac{q-\beta}{\alpha} \in(-q(1-1 / \alpha), 0)$. In this case, $H<0$ and we do not have direct pointwise representation, but the limit field $Z_{\alpha, \beta}^{E}$ can be obtained as the derivative (in the sense of distributions) of a pointwise process. For all $t \in \mathbb{R}^{d}$, following the same idea as for the definition of $Z_{\alpha, \beta}^{E}(f)$ for $f \in \mathcal{S}\left(\mathbb{R}^{d}\right)$, we can define the random variable

$$
\check{Z}_{\alpha, \beta}^{E}(t)=\epsilon\left(t_{1}\right) \cdots \epsilon\left(t_{d}\right) \int_{\mathbb{R}^{d} \times \mathbb{R}_{+}} T_{r}^{E} \mathbf{1}_{[0, t]}(x) M_{\alpha, \beta}(d r, d x),
$$

where the random measure $M_{\alpha, \beta}$ is the same as in $(3.2),[0, t]=\prod_{i=1}^{d}\left[0, t_{i}\right]$, and $\epsilon\left(t_{i}\right)$ is the sign of $t_{i}$. The family $\breve{Z}_{\alpha, \beta}^{E}=\left\{\check{Z}_{\alpha, \beta}^{E}(t)\right\}_{t \in \mathbb{R}^{d}}$ is a measurable random field and, by successive integrations by parts, we can show that $Z_{\alpha, \beta}^{E}=D^{(1, \ldots, 1)} \check{Z}_{\alpha, \beta}^{E}$, that is for all $f \in \mathcal{S}\left(\mathbb{R}^{d}\right)$,

$$
Z_{\alpha, \beta}^{E}(f)=(-1)^{d} \int_{\mathbb{R}^{d}} \check{Z}_{\alpha, \beta}^{E}(t) D^{(1, \ldots, 1)} f(t) d t .
$$

This consideration is analogous to Theorem 2.6 and Lemma 3.7 in Breton and Dombry (2011) for $E=I_{d}$ and $\beta>q=d$ in $\mathcal{D}^{\prime}\left(\mathbb{R}^{d}\right)$ the space of distribution instead of $\mathcal{S}^{\prime}\left(\mathbb{R}^{d}\right)$. We thus refer to Breton and Dombry (2011) for technical details.

\section{Proofs of the main results}

6.1. Preliminary results. The proofs of our limit theorems follow the same scheme as in Biermé et al. (2010) or Breton and Dombry (2009) to establish the convergence of the characteristic functions. They use the two following lemmas concerning conditions (2.7) and (2.8).

Lemma 6.1 (Lemma 2.4 in Biermé et al., 2010, Lemma 3.2 in Breton and Dombry, 2009). Under the assumption (2.7), if $\left\{g_{\rho}\right\}_{\rho>0}, g$ are continuous functions on $\mathbb{R}_{+}$ such that

$$
\lim _{\rho \rightarrow 0^{\beta-q}}\left|g(r)-g_{\rho}(r)\right|=0,
$$

and for some $0<\beta_{-}<\beta<\beta_{+}$there exists a constant $C>0$ such that

$$
\begin{aligned}
& |g(r)| \leqslant C\left(r^{\beta_{-}} \wedge r^{\beta_{+}}\right), \\
& \left|g_{\rho}(r)\right| \leqslant C\left(r^{\beta_{-}} \wedge r^{\beta_{+}}\right),
\end{aligned}
$$

for all $r>0$, then, for $C_{\beta}$ as in (2.7),

$$
\int_{\mathbb{R}_{+}} g_{\rho}(r) F_{\rho}(d r) \sim C_{\beta} \rho^{\beta} \int_{\mathbb{R}_{+}} g(r) r^{-1-\beta} d r, \quad \text { as } \rho \rightarrow 0^{\beta-q} .
$$


Lemma 6.2 (Lemma 3.1 in Breton and Dombry, 2009). Suppose that $M$ is in the domain of attraction of $S_{\alpha}(\sigma, b, 0)$ for some $\alpha>1, \sigma>0$ and $b \in \mathbb{R}$. Then

$$
\phi_{G}(t)=\mathcal{L}_{M}(t)-1-i t \mathbb{E}(M) \sim-|t|^{\alpha} \phi_{\alpha, b, \sigma}(t) \text {, as } t \rightarrow 0,
$$

with

$$
\phi_{\alpha, b, \sigma}(t)=\sigma^{\alpha}(1-i b \epsilon(t) \tan (\alpha \pi / 2)),
$$

where $\epsilon(t)=\operatorname{sign}(t)$. Furthermore, there exists $C>0$ such that for all $t \in \mathbb{R}$,

$$
\left|\phi_{G}(t)\right| \leqslant C|t|^{\alpha} \text {. }
$$

The key ingredients for our generalized random ball model are the precise continuity properties of the operators $T_{r}^{E}$ stated in the following proposition. Recall that we write $v_{r}=\operatorname{Leb}_{d}\left(B_{E}(0, r)\right)=r^{q} v_{B}, r>0$, and for $\gamma>0,\|f\|_{L^{\gamma}}^{\gamma}=\int_{\mathbb{R}^{d}}|f(x)|^{\gamma} d x$.

Proposition 6.3. (i) For all $\gamma \in[1,2], r>0$, and $f \in \mathcal{S}\left(\mathbb{R}^{d}\right)$,

$$
\left\|T_{r}^{E} f\right\|_{L^{\gamma}} \leqslant v_{r}\|f\|_{L^{\gamma}}
$$

and

$$
\left\|T_{r}^{E} f\right\|_{L^{\gamma}} \leqslant v_{r}^{1 / \gamma}\|f\|_{L^{1}} .
$$

As a consequence, for $\gamma \in(1,2]$ and $\beta \in(q, \gamma q)$, there exists some constant $C>0$ such that

$$
\int_{\mathbb{R}_{+}}\left\|T_{r}^{E} f\right\|_{L^{\gamma}}^{\gamma} r^{-1-\beta} d r \leqslant C\|f\|_{L^{1} \cap L^{\gamma}}^{\gamma}, \quad f \in \mathcal{S}\left(\mathbb{R}^{d}\right),
$$

with $\|f\|_{L^{1} \cap L^{\gamma}}:=\|f\|_{L^{1}} \vee\|f\|_{L^{\gamma}}$.

(ii) For all $\gamma \in[1,2], r>1$, and $f \in \mathcal{S}_{1}\left(\mathbb{R}^{d}\right)$,

$$
\left\|T_{r}^{E} f\right\|_{L^{\gamma}}^{\gamma} \leqslant C r^{q-a_{d}}(|\log r| \vee 1)^{\ell_{d}-1}\|f\|_{L^{1}}^{\gamma-1} \int_{\mathbb{R}^{d}}|y \| f(y)| d y,
$$

where $\ell_{d} \leqslant d$ is the number of eigenvalues of $E$ having the minimal real part $a_{d}$ (counted with multiplicities). As a consequence, for $\beta \in\left(q-a_{d}, q\right)$ there exists a constant $C$ such that

$$
\int_{\mathbb{R}_{+}}\left\|T_{r}^{E} f\right\|_{L^{\gamma}}^{\gamma} r^{-1-\beta} d r \leqslant C\|f\|_{L^{1}}^{\gamma-1} \int_{\mathbb{R}^{d}}(1+|y|)|f(y)| d y, \quad f \in \mathcal{S}_{1}\left(\mathbb{R}^{d}\right) .
$$

Proof: (i) Note that

$$
\left\|T_{r}^{E} f\right\|_{L^{1}}:=\int_{\mathbb{R}^{d}}\left|T_{r}^{E} f(x)\right| d x \leqslant \int_{\mathbb{R}^{d}} \int_{\mathbb{R}^{d}} K_{r}^{E}(x, y)|f(y)| d y d x,
$$

with $K_{r}^{E}(x, y)=\mathbf{1}_{B_{E}(x, r)}(y)$ by (2.2). Hence, by Fubini's theorem,

$$
\left\|T_{r}^{E} f\right\|_{L^{1}} \leqslant v_{r}\|f\|_{L^{1}} .
$$

Moreover,

$$
\left\|T_{r}^{E} f\right\|_{L^{2}}^{2}=\int_{\mathbb{R}^{d}}\left|T_{r}^{E} f(x)\right|^{2} d x \leqslant \int_{\mathbb{R}^{d}} v_{r} \int_{\mathbb{R}^{d}} K_{r}^{E}(x, y)|f(y)|^{2} d y d x=v_{r}^{2}\|f\|_{L^{2}}^{2},
$$

where we first applied the Cauchy-Schwarz inequality, and Fubini's theorem at the end. According to the Riesz-Thorin interpolation theorem (see Bergh and Löfström (1976)), combining this with (6.11), we get (6.6). Moreover, since by the CauchySchwarz inequality we also have

$$
\left\|T_{r}^{E} f\right\|_{L^{2}}^{2} \leqslant \int_{\mathbb{R}^{d}} \int_{\mathbb{R}^{d}} K_{r}^{E}(x, y)|f(y)| d y\|f\|_{L^{1}} d x=v_{r}\|f\|_{L^{1}}^{2},
$$


it follows by Hölder's inequality that, for $p>1$ such that $\gamma=1 / p+2(1-1 / p)$,

$$
\left\|T_{r}^{E} f\right\|_{L^{\gamma}}^{\gamma} \leqslant\left\|T_{r}^{E} f\right\|_{L^{1}}^{1 / p}\left\|T_{r}^{E} f\right\|_{L^{2}}^{2(1-1 / p)} \leqslant v_{r}^{1 / p}\|f\|_{L^{1}}^{1 / p} v_{r}^{1-1 / p}\|f\|_{L^{1}}^{2(1-1 / p)}=v_{r}\|f\|_{L^{1}}^{\gamma} .
$$

Since $v_{r}=r^{q} v_{B}$ with $q=\operatorname{tr}(E)$ we can conclude that for $\beta \in(q, \gamma q)$, by (6.6) and (6.7),

$$
\int_{\mathbb{R}_{+}}\left\|T_{r}^{E} f\right\|_{L^{\gamma}}^{\gamma} r^{-\beta-1} d r \leqslant\left(\left(v_{B}\|f\|_{L^{1}}^{\gamma}\right) \vee\left(v_{B}^{\gamma}\|f\|_{L^{\gamma}}^{\gamma}\right)\right) \int_{\mathbb{R}_{+}} r^{q-\beta-1} \wedge r^{\gamma q-\beta-1} d r .
$$

Therefore we have proved (6.8).

(ii) The assumption that $f \in \mathcal{S}_{1}\left(\mathbb{R}^{d}\right)$ implies that $\int_{\mathbb{R}^{d}} f(z) d z=0$ so that

$$
T_{r}^{E} f(x)=\int_{\mathbb{R}^{d}} \tilde{K}_{r}^{E}(x, y) f(y) d y,
$$

with $\tilde{K}_{r}^{E}(x, y)=\mathbf{1}_{B_{E}(x, r)}(y)-\mathbf{1}_{B_{E}(x, r)}(0)$. Then, by Hölder's inequality, one has

$$
\begin{aligned}
\left\|T_{r}^{E} f\right\|_{L^{\gamma}}^{\gamma} & =\int_{\mathbb{R}^{d}}\left|\int_{\mathbb{R}^{d}}\left(\mathbf{1}_{B_{E}(y, r)}(x)-\mathbf{1}_{B_{E}(0, r)}(x)\right) f(y) d y\right|^{\gamma} d x \\
& \leqslant\|f\|_{L^{1}}^{\gamma-1} \int_{\mathbb{R}^{d}}\left(\int_{\mathbb{R}^{d}}\left|\mathbf{1}_{B_{E}(y, r)}(x)-\mathbf{1}_{B_{E}(0, r)}(x)\right|^{\gamma}|f(y)| d y\right) d x .
\end{aligned}
$$

Also,

$$
\int_{\mathbb{R}^{d}}\left|\mathbf{1}_{B_{E}(y, r)}(x)-\mathbf{1}_{B_{E}(0, r)}(x)\right|^{\gamma} d x=\operatorname{Leb}_{d}\left(B_{E}(y, r) \triangle B_{E}(0, r)\right)=r^{q} h\left(r^{-E} y\right)
$$

with $h(z)=\operatorname{Leb}_{d}\left(B_{E}(0,1) \triangle B_{E}(z, 1)\right)=\operatorname{Leb}_{d}(B \triangle(z+B))$, that does not depend on $E$. By $(2.4), h(y) \leqslant C|y|$ for all $y \in \mathbb{R}^{d}$ and it follows that,

$$
\left\|T_{r}^{E} f\right\|_{L^{\gamma}}^{\gamma} \leqslant C\|f\|_{L^{1}}^{\gamma-1} \int_{\mathbb{R}^{d}} r^{q}\left|r^{-E} y \| f(y)\right| d y .
$$

Recall that according to the Jordan decomposition theorem, given $E$, there exists an invertible matrix $P$ such that $D=P^{-1} E P$ has the real canonical form

$$
\left(\begin{array}{ccc}
J_{1} & & 0 \\
& \ddots & \\
0 & & J_{p}
\end{array}\right),
$$

where $p$ corresponds to the number of distinct real parts of eigenvalues and each block matrix $J$ is either

(i) a Jordan cell matrix of size $\ell$

$$
\left(\begin{array}{cccc}
a & 0 & & 0 \\
1 & a & \ddots & \\
& \ddots & \ddots & 0 \\
0 & & 1 & a
\end{array}\right),
$$

with $a$ a real eigenvalue of $E$, or 
(ii) a $2 \ell \times 2 \ell$ matrix in form of

$$
\left(\begin{array}{cccc}
\Lambda & & & 0 \\
I_{2} & \Lambda & & \\
& \ddots & \ddots & \\
0 & & I_{2} & \Lambda
\end{array}\right) \quad \text { with } \Lambda=\left(\begin{array}{cc}
a & b \\
b & a
\end{array}\right) \text { and } I_{2}=\left(\begin{array}{ll}
1 & 0 \\
0 & 1
\end{array}\right) \text {, }
$$

with $a \pm i b(b \neq 0)$ being complex conjugated eigenvalues of $E$.

In either case, for the subordinated norm $\|\cdot\|$ of the Euclidean norm on $\mathbb{R}^{d}$, for each block $J$ with the corresponding real part of eigenvalue denoted by $a$, it is shown in Lemma 3.2 of Biermé and Lacaux (2009) that

$$
r^{a} \leqslant\left\|r^{J}\right\| \leqslant \sqrt{2 \ell} e r^{a}(|\log r| \vee 1)^{\ell-1}, \text { for all } r>0 .
$$

(This is slightly different from Lemma 3.2 in Biermé and Lacaux, 2009, but can be easily established by following the proof carefully.) Recall that it is assumed that the real parts of eigenvalues of $E$ satisfy $a_{1} \geqslant \cdots \geqslant a_{d}>0$. Let $\ell_{d}$ be the size of the Jordan block associated with $a_{d}$ and note that the other Jordan blocks, if they exist, are associated with a strictly greater real part. Then, there exists a constant $C>0$, such that

$$
\left\|r^{E}\right\| \leqslant C r^{a_{d}}(|\log r| \vee 1)^{\ell_{d}-1}, \text { for all } r \in(0,1) .
$$

Now, it follows from (6.12) that for $f \in \mathcal{S}_{1}\left(\mathbb{R}^{d}\right)$ one has for $r>1$,

$$
\left\|T_{r}^{E} f\right\|_{L^{\gamma}}^{\gamma} \leqslant C r^{q-a_{d}}(|\log r| \vee 1)^{\ell_{d}-1}\|f\|_{L^{1}}^{\gamma-1} \int_{\mathbb{R}^{d}}|y f(y)| d y .
$$

Hence, for $\beta \in\left(q-a_{d}, q\right), f \in \mathcal{S}_{1}\left(\mathbb{R}^{d}\right)$, combining the above inequality for $r>1$ with (6.7) for $r \leqslant 1$, we obtain

$$
\begin{aligned}
\int_{\mathbb{R}_{+}}\left\|T_{r}^{E} f\right\|_{L^{\gamma}}^{\gamma} r^{-1-\beta} d r \leqslant & C\left(\|f\|_{L^{1}}^{\gamma-1} \int_{\mathbb{R}^{d}}(1+|y|)|f(y)| d y\right) \\
& \times \int_{\mathbb{R}_{+}} r^{-1-\beta+q} \wedge\left(r^{-1-\beta+q-a_{d}}(|\log r| \vee 1)^{\ell_{d}-1}\right) d r
\end{aligned}
$$

which proves (6.10).

\subsection{Dense regime.}

Proof of Proposition 3.1: First, the stochastic integral $Z_{\alpha, \beta}^{E}(f)$ in (3.2) is welldefined as soon as

$$
\int_{\mathbb{R}^{d} \times \mathbb{R}_{+}}\left|T_{r}^{E} f(x)\right|^{\alpha} r^{-1-\beta} d r d x=\int_{\mathbb{R}_{+}}\left\|T_{r}^{E} f\right\|_{L^{\alpha}}^{\alpha} r^{-1-\beta} d r<\infty
$$

and this condition follows from Proposition 6.3, with $\gamma=\alpha, \beta, n$ as in (3.1). It is well known (see Samorodnitsky and Taqqu, 1994, Chap. 3) that the characteristic functional $\mathcal{L}_{Z_{\alpha, \beta}^{E}}$ of $Z_{\alpha, \beta}^{E}$ on $\mathcal{S}_{n}\left(\mathbb{R}^{d}\right)$ is given by (3.3). Now, according to Theorem 2.1, to prove the existence of a generalized-random-field version of $Z_{\alpha, \beta}^{E}$, it suffices to prove that $\mathcal{L}_{Z_{\alpha, \beta}^{E}}$ is continuous on $\mathcal{S}_{n}\left(\mathbb{R}^{d}\right)$, that is, for all $\left\{f_{k}\right\}_{k \in \mathbb{N}}$ and $f$ in $\mathcal{S}_{n}\left(\mathbb{R}^{d}\right)$ such that $f_{k} \rightarrow f$ in $\mathcal{S}_{n}\left(\mathbb{R}^{d}\right), \lim _{k \rightarrow \infty} \mathcal{L}_{Z_{\alpha, \beta}^{E}}\left(f_{k}\right)=\mathcal{L}_{Z_{\alpha, \beta}^{E}}(f)$. This shall 
follow from the convergence in distribution of the random variables $Z_{\alpha, \beta}^{E}\left(f_{k}-f\right)$ to 0 as $k \rightarrow \infty$, or equivalently from

$$
\lim _{k \rightarrow \infty} \int_{\mathbb{R}^{d} \times \mathbb{R}_{+}}\left\|T_{r}^{E}\left(f_{k}-f\right)\right\|_{L^{\alpha}}^{\alpha} r^{-1-\beta} d r=0 .
$$

By (6.8) and (6.10) of Proposition 6.3 with $\gamma=\alpha$, this is straightforward, since $f_{k}-f \rightarrow 0$ in $\mathcal{S}_{n}\left(\mathbb{R}^{d}\right)$ clearly implies that the upper bounds also tend to 0 .

Proof of Theorem 3.2: Note that, by Theorem 2.2, the result follows from the pointwise convergence of the characteristic functional. Further, by (2.9), we clearly have for $f \in \mathcal{S}_{n}\left(\mathbb{R}^{d}\right)$,

$$
\mathcal{L}_{n_{1}(\rho)^{-1 / \alpha} Y_{\rho}^{E}}(f)=\exp \left(\int_{\mathbb{R}^{d} \times \mathbb{R}_{+}} \phi_{G}\left(\frac{T_{r}^{E} f(x)}{n_{1}(\rho)^{1 / \alpha}}\right) \lambda(\rho) d x F_{\rho}(d r)\right) .
$$

Since $n_{1}(\rho) \rightarrow \infty$, by Lemma 6.2 ,

$$
\phi_{G}\left(\frac{T_{r}^{E} f(x)}{n_{1}(\rho)^{1 / \alpha}}\right) \sim-\frac{1}{n_{1}(\rho)}\left|T_{r}^{E} f(x)\right|^{\alpha} \phi_{\alpha, b, \sigma}\left(T_{r}^{E} f(x)\right), \quad \text { as } \rho \rightarrow 0^{\beta-q},
$$

for $\phi_{\alpha, b, \sigma}$ defined in (6.4). Hence, under (2.7), one can apply Lemma 6.1 to prove that

$$
\mathcal{L}_{n_{1}(\rho)^{-1 / \alpha} Y_{\rho}^{E}}(f) \rightarrow \mathcal{L}_{Z_{\alpha, \beta}^{E}}(f) .
$$

Indeed, recall the uniform bound (6.5) on $\phi_{G}$ and, thanks to Proposition 6.3, the fact that for $n=0$,

and for $n=1$,

$$
\left\|T_{r}^{E} f\right\|_{L^{\alpha}}^{\alpha} \leqslant C_{E}\|f\|_{L^{1} \cap L^{\alpha}}^{\alpha}\left(r^{q} \wedge r^{\alpha q}\right),
$$

$$
\left\|T_{r}^{E} f\right\|_{L^{\alpha}}^{\alpha} \leqslant C_{E}\|f\|_{L^{1}}^{\alpha-1}\left(\int_{\mathbb{R}^{d}}(1+|y|)|f(y)| d y\right)\left(r^{q} \wedge r^{q-a_{p}}|\log (r)|^{d-1}\right) .
$$

We can then apply Lemma 6.1 with $g_{\rho}(r)=n_{1}(\rho) \int_{\mathbb{R}^{d}} \phi_{G}\left(n_{1}(\rho)^{-1 / \alpha} T_{r}^{E} f(x)\right) d x$ to both cases $\beta \in(q, \alpha q)$ and $\beta \in\left(q-a_{d}, q\right)$.

\subsection{Intermediate regime.}

Proof of Proposition 3.4: Recall that the Poisson integral $J_{a, \alpha, \beta}^{E}(f)$ in (3.6) is welldefined as soon as

$$
\int_{\mathbb{R}^{d} \times \mathbb{R}_{+} \times \mathbb{R}_{+}}\left(\left|m T_{r, a}^{E} f(x)\right| \wedge\left|m T_{r, a}^{E} f(x)\right|^{2}\right) r^{-1-\beta} d x d r G(d m)<\infty .
$$

Let us remark that

$$
\left|m T_{r, a}^{E} f(x)\right| \wedge\left|m T_{r, a}^{E} f(x)\right|^{2} \leqslant\left|m T_{r, a}^{E} f(x)\right|^{\gamma},
$$

for any $\gamma \in[1,2]$. Hence, for $\beta \in\left(q-a_{d}, q\right) \cup(q, \alpha q)$, choosing $\gamma \in[1, \alpha)$ such that $\beta \in\left(q-a_{d}, \gamma q\right)$, one has

$$
\int_{\mathbb{R}^{d} \times \mathbb{R}_{+} \times \mathbb{R}_{+}}\left|m T_{r, a}^{E} f(x)\right|^{\gamma} r^{-1-\beta} d x d r G(d m) \leqslant \mathbb{E}\left(|M|^{\gamma}\right) \int_{\mathbb{R}^{+}}\left\|T_{r, a}^{E} f\right\|_{L^{\gamma}}^{\gamma} r^{-1-\beta} d r<\infty,
$$

in view of Proposition 6.3, since $\left\|T_{r, a}^{E} f\right\|_{L^{\gamma}}^{\gamma}=a^{q}\left\|T_{r / a}^{E} f\right\|_{L^{\gamma}}^{\gamma}$ (see (3.5)). It follows that the Poisson integral $J_{a, \alpha, \beta}^{E}(f)$ is well-defined for all $f \in \mathcal{S}_{n}\left(\mathbb{R}^{d}\right)$ and the characteristic functional $\mathcal{L}_{J_{a, \alpha, \beta}^{E}}$ of $J_{a, \alpha, \beta}^{E}$ is given by (3.7). 
Again, to show the existence of a version of $J_{a, \alpha, \beta}^{E}$ with values in $\mathcal{S}_{n}^{\prime}\left(\mathbb{R}^{d}\right)$, using Theorem 2.1, it is sufficient to prove that the characteristic functional $\mathcal{L}_{J_{a, \alpha, \beta}^{E}}$ is continuous on $\mathcal{S}_{n}\left(\mathbb{R}^{d}\right)$. Let $\beta \in\left(q-a_{d}, q\right) \cup(q, \alpha q)$ and assume that $f_{k} \rightarrow 0$ in $\mathcal{S}_{n}\left(\mathbb{R}^{d}\right)$. We will show that $J_{a, \alpha, \beta}^{E}\left(f_{k}\right)$ converges in $L^{\gamma}$ to 0 , which is sufficient to prove the continuity of $\mathcal{L}_{J_{a, \alpha, \beta}^{E}}$. Actually, following the proof of Proposition 3.1 in Breton and Dombry (2011), we can bound $\gamma$-moments of the real random variable $J_{a, \alpha, \beta}^{E}(f)$ for $f \in \mathcal{S}_{n}\left(\mathbb{R}^{d}\right)$. Since $J_{a, \alpha, \beta}^{E}(f)$ is centered, for $\gamma \in[1, \alpha)$, following Gaigalas (2006) page 461 and using Lemma 2 and Lemma 4 of von Bahr and Esseen (1965),

$$
\mathbb{E}\left(\left|J_{a, \alpha, \beta}^{E}(f)\right|^{\gamma}\right) \leqslant A(\gamma) \int_{0}^{\infty}\left(1-\left|\mathcal{L}_{J_{a, \alpha, \beta}^{E}}(\theta f)\right|^{2}\right) \theta^{-1-\gamma} d \theta,
$$

with $A(\gamma):=\left(\int_{0}^{\infty}(1-\cos x) x^{-1-\gamma} d x\right)^{-1}<\infty$. But

$$
\left|\mathcal{L}_{J_{a, \alpha, \beta}^{E}}(\theta f)\right| \geqslant \exp \left(-C|\theta|^{\alpha} \int_{\mathbb{R}^{d} \times \mathbb{R}^{+}}\left|T_{r, a}^{E} f(x)\right|^{\alpha} C_{\beta} r^{-1-\beta} d r d x\right),
$$

using the upper bound on $\left|\phi_{G}\right|$ given (6.5). It follows that for $\gamma \in[1, \alpha)$ one has

$$
\begin{aligned}
\mathbb{E}\left(\left|J_{a, \alpha, \beta}^{E}(f)\right|^{\gamma}\right) & \leqslant A(\gamma) \int_{0}^{\infty}\left(1-\exp \left(-2 C|\theta|^{\alpha} \int_{\mathbb{R}^{+}}\left\|T_{r, a}^{E} f\right\|_{L^{\alpha}}^{\alpha} C_{\beta} r^{-1-\beta} d r\right) \theta^{-1-\gamma} d \theta\right. \\
& \leqslant A(\gamma) A(\alpha, \gamma)\left(C \int_{\mathbb{R}^{+}}\left\|T_{r, a}^{E} f\right\|_{L^{\alpha}}^{\alpha} C_{\beta} r^{-1-\beta} d r\right)^{\gamma / \alpha}
\end{aligned}
$$

with $A(\alpha, \gamma):=\int_{0}^{\infty}\left(1-\exp \left(-s^{\alpha}\right)\right) s^{-1-\gamma} d s<\infty$. Hence the result follows from Proposition 6.3 since $\left\|T_{r, a}^{E} f\right\|_{L^{\alpha}}^{\alpha}=a^{q}\left\|T_{r / a}^{E} f\right\|_{L^{\alpha}}^{\alpha}$.

Proof of Theorem 3.5: Again, by Theorem 2.2, the result follows from the convergence of the characteristic functionals. Observe that,

$$
\begin{aligned}
\mathcal{L}_{J_{a, \alpha, \beta}^{E}}(f) & =\exp \left\{\int_{\mathbb{R}^{d} \times \mathbb{R}_{+}} \phi_{G}\left(T_{r, a}^{E} f(x)\right) C_{\beta} r^{-1-\beta} d r d x\right\} \\
& =\exp \left\{C_{\beta} \int_{\mathbb{R}^{d} \times \mathbb{R}_{+}} \phi_{G}\left(T_{s}^{E} f(y)\right) a^{q-\beta} s^{-1-\beta} \mathrm{d} s d y\right\}
\end{aligned}
$$

by the changes of variables $y=a^{-E} x$ and $s=r / a$. The rest of the proof can be done similarly as for Theorem 3.2, starting from (2.9) and applying Lemma 6.1 with $g(r)=g_{\rho}(r)=\int_{\mathbb{R}^{d}} \phi_{G}\left(T_{r}^{E} f(x)\right) d x$ and the help of Proposition 6.3.

\subsection{Sparse regime.}

Proof of Proposition 3.6: Using Theorem 2.1, it is sufficient to prove that $Z_{\gamma}^{(1)}\left(f_{k}\right)$ converges in distribution to 0 when $f_{k} \rightarrow 0$ in $\mathcal{S}\left(\mathbb{R}^{d}\right)$. This last assertion is obvious since convergence in $\mathcal{S}\left(\mathbb{R}^{d}\right)$ implies convergence in $L^{\gamma}\left(\mathbb{R}^{d}\right)$.

To prove Theorem 3.7, we consider the maximal function $f^{*}$ associated to a function $f$ of $\mathcal{S}\left(\mathbb{R}^{d}\right)$,

$$
f^{*}(x):=\sup _{r>0} \frac{1}{r^{q} v_{B}} \int \mathbf{1}_{B_{E}(x, r)}(y)|f(y)| d y, \quad x \in \mathbb{R}^{d},
$$

and we shall need the following lemma. 
Lemma 6.4. For all $f \in \mathcal{S}\left(\mathbb{R}^{d}\right)$ and all $\alpha>1, f^{*} \in L^{\alpha}\left(\mathbb{R}^{d}\right)$.

Proof: By Lemma 6.1.5 in Meerschaert and Scheffler (2004), there exists a norm $\|\cdot\|_{0}$ on $\mathbb{R}^{d}$ such that the mapping $(0, \infty) \times\left\{x \in \mathbb{R}^{d} \mid\|x\|_{0}=1\right\} \rightarrow \mathbb{R}^{d} \backslash\{0\}$, $(t, \theta) \mapsto t^{E} \theta$, is a homeomorphism. Further, the function $t \mapsto\left\|t^{E} x\right\|_{0}$ is increasing for all $x \in \mathbb{R}^{d}$. Thus, any $x \in \mathbb{R}^{d} \backslash\{0\}$ can be uniquely written as $x=\tau(x)^{E} \theta(x)$ with $\tau(x)>0$ and $\|\theta(x)\|_{0}=1$. The function $\tau$ is a continuous function that can be extended to $\mathbb{R}^{d}$ by setting $\tau(0)=0$. By Lemma 2.2 in Biermé et al. (2007), one can find $\kappa \geqslant 1$ such that

$$
\tau(x+y) \leqslant \kappa(\tau(x)+\tau(y)) .
$$

Therefore we can introduce the function $\delta(x, y)=\tau(y-x), x, y \in \mathbb{R}^{d}$, which is a quasi-distance on $\mathbb{R}^{d}$. We also introduce the sets

$$
C_{E}(x, r)=\left\{y \in \mathbb{R}^{d} \mid \delta(x, y)<r\right\}, \quad r>0 .
$$

Since $B$ is a bounded subset of $\mathbb{R}^{d}$, we can find a real $r_{0}>0$ such that $B \subset C_{E}\left(0, r_{0}\right)$. With no loss of generality we assume that $r_{0}=1$ and we denote $C:=C_{E}(0,1)$. Thus $C_{E}(x, r)=x+r^{E} C$ for all $x \in \mathbb{R}^{d}$ and $r>0$, and $B_{E}(x, r) \subset C_{E}(x, r)$. We infer that for all $x \in \mathbb{R}^{d}$,

$$
f^{*}(x) \leqslant \frac{v_{C}}{v_{B}} \sup _{r>0} \frac{1}{r^{q} v_{C}} \int \mathbf{1}_{C_{E}(x, r)}(y)|f(y)| d y .
$$

The desired result is now a consequence of Theorem 1 and Example 2.4 in Stein (1993).

Proof of Theorem 3.7: By Theorem 2.2, it is sufficient to prove the convergence of the characteristic functionals. The characteristic functional of $n_{2}(\rho)^{-1} Y_{\rho}^{E}$ is given by, recalling that $F_{\rho}(d r)=F(d r / \rho)$,

$$
\begin{aligned}
\mathcal{L}_{n_{2}(\rho)^{-1} Y_{\rho}^{E}}(f) & =\exp \left\{\int_{\mathbb{R}^{d} \times \mathbb{R}_{+}} \lambda(\rho) \phi_{G}\left(\frac{T_{r}^{E} f(x)}{n_{2}(\rho)}\right) d x F_{\rho}(d r)\right\} \\
& =\exp \left\{\int_{\mathbb{R}^{d} \times \mathbb{R}_{+}} \lambda(\rho) \phi_{G}\left(\frac{T_{n_{2}(\rho)^{1 / q} r}^{E} f(x)}{n_{2}(\rho)}\right) d x F_{\rho n_{2}(\rho)^{-1 / q}}(d r)\right\} .
\end{aligned}
$$

We shall show that

$$
\begin{aligned}
& \int_{\mathbb{R}^{d} \times \mathbb{R}_{+}} \lambda(\rho) \phi_{G}\left(\frac{T_{n_{2}(\rho)^{1 / q} r}^{E} f(x)}{n_{2}(\rho)}\right) d x F_{\rho n_{2}(\rho)^{-1 / q}}(d r) \\
& \rightarrow C_{\beta} \int_{\mathbb{R}^{d} \times \mathbb{R}_{+}} \phi_{G}\left(f(x) v_{B} r^{q}\right) r^{-1-\beta} d r d x \quad \text { as } \rho \rightarrow 0^{\beta-q} \text { and } \lambda(\rho) \rightarrow 0^{q-\beta} .
\end{aligned}
$$

From this, we infer that

$$
\mathcal{L}_{n_{2}(\rho)^{-1} Y_{\rho}^{E}}(f) \rightarrow \exp \left\{C_{\beta} \int_{\mathbb{R}^{d} \times \mathbb{R}_{+}} \phi_{G}\left(f(x) v_{B} r^{q}\right) r^{-1-\beta} d r d x\right\}=\mathcal{L}_{Z_{\gamma}^{(1)}}(f),
$$

for $\mathcal{L}_{Z_{\gamma}^{(1)}}(f)$ given in (3.10), which completes the proof. The last equality above is obtained by following the same lines as in Breton and Dombry (2009) pages $3650-3651$. 
To prove (6.15), recalling that $\lambda(\rho) \rho^{\beta} n_{2}(\rho)^{-\beta / q}=1$, it suffices to check the conditions of Lemma 6.1 for

$$
g_{\rho}(r):=\int_{\mathbb{R}^{d}} \phi_{G}\left(\frac{T_{n_{2}(\rho)^{1 / q} r}^{E} f(x)}{n_{2}(\rho)}\right) d x \quad \text { and } \quad g(r):=\int_{\mathbb{R}^{d}} \phi_{G}\left(f(x) v_{B} r^{q}\right) d x .
$$

First, remark that for $f \in \mathcal{S}_{n}\left(\mathbb{R}^{d}\right)$,

$$
\frac{T_{n_{2}(\rho)^{1 / q} r}^{E} f(x)}{n_{2}(\rho)} \underset{\rho \rightarrow 0^{\beta-q}}{\longrightarrow} v_{B} r^{q} f(x)
$$

for $d x$-almost all $x$, so that

$$
\phi_{G}\left(\frac{T_{n_{2}(\rho)^{1 / q} r}^{E} f(x)}{n_{2}(\rho)}\right) \underset{\rho \rightarrow 0^{-1}-q}{\longrightarrow} \phi_{G}\left(v_{B} r^{q} f(x)\right)
$$

for $d x$-almost all $x$ by continuity of $\phi_{G}$. But, by Lemma 6.2 ,

$$
\left|\phi_{G}\left(\frac{T_{n_{2}(\rho)^{1 / q} r}^{E} f(x)}{n_{2}(\rho)}\right)\right| \leqslant C\left|\frac{T_{n_{2}(\rho)^{1 / q} r}^{E} f(x)}{n_{2}(\rho)}\right|^{\alpha} \leqslant C\left(v_{B} r^{q}\right)^{\alpha} f^{*}(x)^{\alpha} .
$$

Since $f^{*}$ belongs to $L^{\alpha}\left(\mathbb{R}^{d}\right)$ by Lemma 6.4, Condition (6.1) follows by Lebesgue's theorem.

Next, for Condition (6.2), we deal with the cases $n=0$ and $n=1$ separately. Now, since $\left|\phi_{G}(u)\right| \leqslant C\left(|u| \wedge|u|^{\alpha}\right)$ and $f \in L^{1}\left(\mathbb{R}^{d}\right) \cap L^{\alpha}\left(\mathbb{R}^{d}\right)$,

$$
|g(r)| \leqslant C \int_{\mathbb{R}^{d}}\left|f(x) v_{B} r^{q}\right| \wedge\left|f(x) v_{B} r^{q}\right|^{\alpha} d x \leqslant C\left(\|f\|_{L^{1}} v_{B} \vee\|f\|_{L^{\alpha}}^{\alpha} v_{B}^{\alpha}\right)\left(r^{q} \wedge r^{\alpha q}\right) .
$$

This establishes Condition (6.2) for $\beta \in(q, \alpha q)$ and $n=0$ with $\beta_{-}=q$ and $\beta_{+}=\alpha q$. Next, when $f \in \mathcal{S}_{1}(\mathbb{R})$, remark that

$$
g(r)=\int_{\mathbb{R}^{d}} \phi_{G}\left(f(x) v_{B} r^{q}\right) d x=\int_{\mathbb{R}^{d}} \tilde{\phi}_{G}\left(f(x) v_{B} r^{q}\right) d x,
$$

with $\tilde{\phi}_{G}(u)=\int\left(e^{i m u}-1\right) G(d m)$ so that now $\left|\tilde{\phi}_{G}(u)\right| \leqslant C\left(1 \wedge|u|^{\delta}\right)$ for any $\delta \in(0,1]$. Hence

$$
|g(r)| \leqslant C v_{B}^{\delta}\|f\|_{L^{\delta}}^{\delta} r^{q \delta} .
$$

Choosing $\delta=q /\left(q+a_{d}\right) \in(0,1)$ and $\delta=1$ respectively, we infer that for $n=1$, Condition (6.2) holds for $\beta \in\left(q^{2} /\left(q+a_{d}\right), q\right)$ with $\beta_{-}=q^{2} /\left(q+a_{d}\right)$ and $\beta_{+}=q$, respectively.

It remains to prove that (6.3) holds. We first consider $\beta \in(q, \alpha q)$. Using $\left|\phi_{G}(u)\right| \leqslant C|u|$ and (6.7),

$$
\left|g_{\rho}(r)\right| \leqslant C \frac{1}{n_{2}(\rho)}\left\|T_{n_{2}(\rho)^{1 / q_{r}}}^{E} f\right\|_{L^{1}} \leqslant C\|f\|_{L^{1}} r^{q} .
$$

Then, using $\left|\phi_{G}(u)\right| \leqslant C|u|^{\alpha}$, we can write

$$
\left|g_{\rho}(r)\right| \leqslant C r^{\alpha q} \int_{\mathbb{R}^{d}}\left|\frac{T_{n_{2}(\rho)^{1 / q} r}^{E} f(x)}{n_{2}(\rho) r^{q}}\right|^{\alpha} d x \leqslant C\left\|f^{*}\right\|_{L^{\alpha}}^{\alpha} r^{\alpha q},
$$


that finishes to prove (6.3) when $\beta \in(q, \alpha q)$. Finally, when $\beta \in\left(q^{2} /\left(q+a_{d}\right), q\right)$ and $f \in \mathcal{S}_{1}\left(\mathbb{R}^{d}\right)$, we write $g_{\rho}=g_{\rho}^{(1)}+g_{\rho}^{(2)}$, with

$$
g_{\rho}^{(1)}:=\int_{\tau(x) \leqslant 2 \kappa n_{2}(\rho)^{1 / q_{r}}} \tilde{\phi}_{G}\left(\frac{T_{n_{2}(\rho)^{1 / q} r}^{E} f(x)}{n_{2}(\rho)}\right) d x
$$

and

$$
g_{\rho}^{(2)}:=\int_{\tau(x)>2 \kappa n_{2}(\rho)^{1 / q_{r}}} \tilde{\phi}_{G}\left(\frac{T_{n_{2}(\rho)^{1 / q_{r}}}^{E} f(x)}{n_{2}(\rho)}\right) d x
$$

where $\kappa \geqslant 1$ comes from the quasi-triangular inequality given in (6.13). With this choice we may write for any $z \in\left(n_{2}(\rho)^{1 / q} r\right)^{E} B$,

$$
\tau(x) \leqslant \kappa(\tau(x+z)+\tau(z)) \leqslant \kappa\left(\tau(x+z)+n_{2}(\rho)^{1 / q} r\right),
$$

where, with no loss of generality, we have again assumed that $B \subset C_{E}(0,1)$ (recall (6.14)). It follows that $\tau(x+z)>\frac{1}{2 \kappa} \tau(x)$ for any $z \in\left(n_{2}(\rho)^{1 / q} r\right)^{E} B$ and $x$ such that $\tau(x)>2 \kappa n_{2}(\rho)^{1 / q} r$. Since $f$ is rapidly decreasing, we get for $N \geqslant 1$,

$$
\begin{aligned}
\left|\frac{1}{n_{2}(\rho)} T_{n_{2}(\rho)^{1 / q} r}^{E} f(x)\right| & \leqslant \int_{\mathbb{R}^{d}} \mathbf{1}_{r^{E} B}(z)\left|f\left(x+n_{2}(\rho)^{E / q} z\right)\right| d z \\
& \leqslant C \int_{\mathbb{R}^{d}} \mathbf{1}_{r^{E} B}(z)\left(1+\tau\left(x+n_{2}(\rho)^{E / q} z\right)\right)^{-N} d z \\
& \leqslant C v_{B} r^{q}(1+\tau(x))^{-N},
\end{aligned}
$$

where here and below, the constant $C=C(f)$ does not depend on $r$ and $\rho$. Using that $\left|\tilde{\phi}_{G}(u)\right| \leqslant C|u|^{\delta}$ for $\delta \in(0,1]$, choosing $N=N(\delta, q)$ such that $N \delta>q+1$, it follows that

$$
\left|g_{\rho}^{(2)}(r)\right| \leqslant C r^{q \delta} \int_{\mathbb{R}^{d}}(1+\tau(x))^{-N \delta} d x \leqslant C r^{q \delta} .
$$

Moreover,

$$
\begin{aligned}
\left|g_{\rho}^{(1)}(r)\right| & \leqslant C n_{2}(\rho)^{-\delta} \int_{\tau(x) \leqslant C n_{2}(\rho)^{1 / q} r}\left|T_{n_{2}(\rho)^{1 / q} r}^{E} f(x)\right|^{\delta} d x \\
& \leqslant C n_{2}(\rho)^{-\delta}\left\|T_{n_{2}(\rho)^{1 / q} r}^{E} f\right\|_{L^{p \delta}}^{\delta}\left(n_{2}(\rho) r^{q}\right)^{1-1 / p}
\end{aligned}
$$

by Hölder's inequality for $p>1$. When $n_{2}(\rho)^{1 / q} r \leqslant 1$, we use (6.7) with $p \delta \in[1,2]$. It follows that

$$
\left|g_{\rho}^{(1)}(r)\right| \leqslant C n_{2}(\rho)^{-\delta}\left(n_{2}(\rho) r^{q}\right)^{1 / p} \times\left(n_{2}(\rho) r^{q}\right)^{1-1 / p} \leqslant C n_{2}(\rho)^{1-\delta} r^{q} \leqslant C r^{q \delta},
$$

since $n_{2}(\rho) \leqslant r^{-q}$. When $n_{2}(\rho)^{1 / q} r>1$, we use (6.9) for $p \delta \in[1,2]$. By the assumption that $\beta>q^{2} /\left(q+a_{d}\right)$, we can choose $b \in\left(0, a_{d}\right)$ such that $\beta>q^{2} /(q+b)$ and

$$
\left\|T_{n_{2}(\rho)^{1 / q} r}^{E} f\right\|_{L^{p \delta}}^{\delta} \leqslant C\left(n_{2}(\rho)^{1 / q} r\right)^{(q-b) / p},
$$

by (6.9) since $b<a_{d}$. Hence,

$$
\left|g_{\rho}^{(1)}(r)\right| \leqslant C n_{2}(\rho)^{-\delta+1-b / q p} r^{q-b / p} .
$$

Now we can choose $\delta=q /(q+b) \in(0,1)$ and $p=(1+b / q)>1$ such that $\delta p=1$ and

$$
\left|g_{\rho}^{(1)}(r)\right| \leqslant C r^{q-b /(1+b / q)}=C r^{q^{2} /(q+b)} .
$$


Combining with the previous bounds (6.17) and (6.18) for the same $\delta=q /(q+b)$, we get

$$
\left|g_{\rho}(r)\right| \leqslant C r^{q^{2} /(q+b)}
$$

and we have that (6.3) holds with $\beta_{-}=q^{2} /(q+b)$ and $\beta_{+}=q$ (which we have shown in (6.16) when considering the case $\beta \in(q, \alpha q))$. We have thus proved (6.15) and the theorem.

6.5. Very-sparse regime. Proposition 3.9 can be obtained as before using Theorem 2.1. The proof of Theorem 3.10 is similar to the one of Theorem 3.7 (see also the proof of Theorem 2.19 in Breton and Dombry, 2009). The details of this part are thus omitted.

\section{Appendix A. Illustrations}

We provide several simulations of our operator-scaling random ball model, obtained by following similar ideas as in Biermé et al. (2013). For the sake of simplicity we choose $E=\operatorname{diag}\left(a_{1}, a_{2}\right)$ with $a_{1} \geqslant a_{2}:=1$ and $\beta \in\left(q-a_{d}, q\right)=\left(a_{1}, a_{1}+1\right)$.

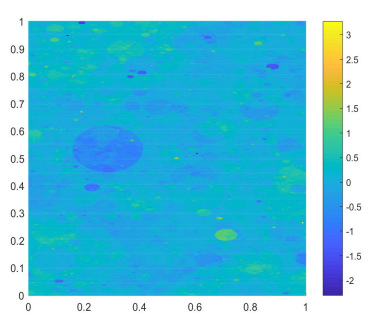

$\alpha=1.7$

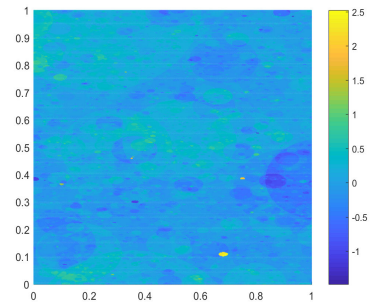

$\alpha=1.9$

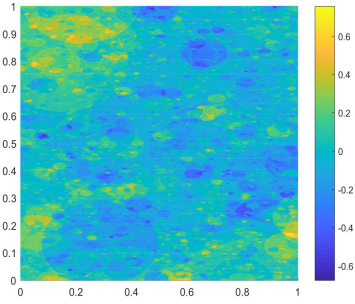

$\alpha=2$

FiguRE A.1. Operator-scaling random ball with $a_{1}=1.2$ and $\beta=1.6$ : the set $B$ is an Euclidean ball, the weights vary according to a $S \alpha S(\sigma)$ distribution with $\sigma=0.1$.
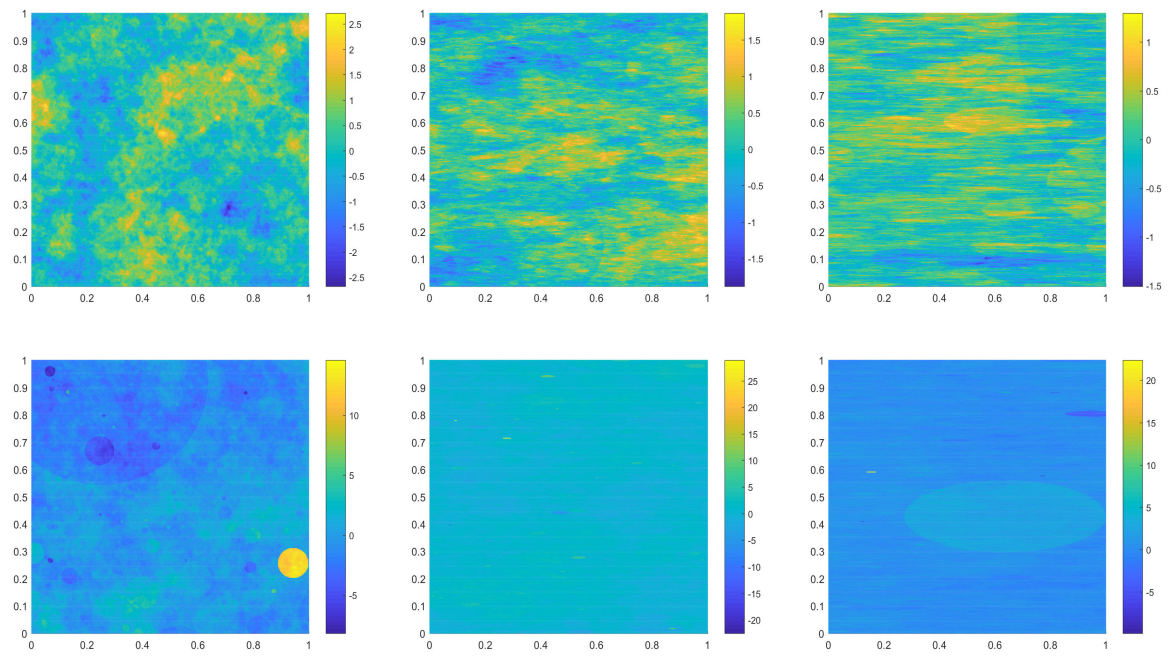

$a_{1}=1$ (isotropic)

$a_{1}=1.5$

$a_{1}=2$

FiguRE A.2. Operator-scaling random ball in high intensity with $H=\frac{1+a_{1}-\beta}{\alpha}=0.4$ and weights following a $S \alpha S(\sigma)$ distribution with $\sigma=0.1$. Top: $\alpha=2$ (Gaussian case). Bottom: $\alpha=1.8$. 

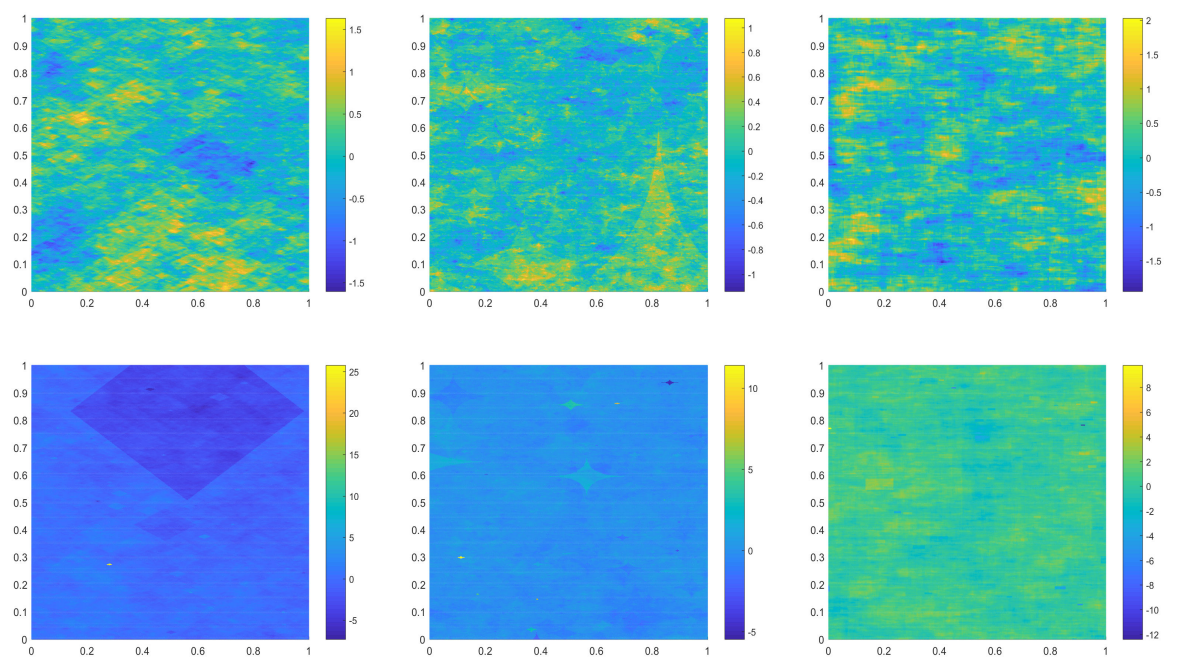

$B_{1}$

$B_{1 / 2}$

$B_{\infty}$

Figure A.3. Operator-scaling random ball in high intensity with $H=\frac{1+a_{1}-\beta}{\alpha}=0.3, a_{1}=1.3$, weights following a $S \alpha S(\sigma)$ distribution with $\sigma=0.1$, and different balls: $B_{1}=\left\{x \in \mathbb{R}^{2}:\left|x_{1}\right|+\left|x_{2}\right| \leqslant 1\right\}, B_{1 / 2}=$ $\left\{x \in \mathbb{R}^{2}:\left|x_{1}\right|^{1 / 2}+\left|x_{2}\right|^{1 / 2} \leqslant 1\right\}$ and $B_{\infty}=\left\{x \in \mathbb{R}^{2}: \max \left(\left|x_{1}\right|,\left|x_{2}\right|\right) \leqslant 1\right\}$. Top: $\alpha=2$ (Gaussian case). Bottom: $\alpha=1.9$.

\section{References}

B. von Bahr and C.-G. Esseen. Inequalities for the $r$ th absolute moment of a sum of random variables, $1 \leqslant r \leqslant 2$. Ann. Math. Statist 36, 299-303 (1965). MR0170407.

J. Beran, Y. Feng, S. Ghosh and R. Kulik. Long-memory processes. Springer, Heidelberg (2013). ISBN 978-3-642-35511-0; 978-3-642-35512-7. MR3075595.

J. Bergh and J. Löfström. Interpolation spaces. An introduction. Springer-Verlag, Berlin-New York (1976). MR0482275.

H. Biermé, Y. Demichel and A. Estrade. Fractional Poisson field and fractional Brownian field: why are they resembling but different? Electron. Commun. Probab. 18, no. 11, 13 (2013). MR3033594.

H. Biermé, O. Durieu and Y. Wang. Generalized random fields and Lévy's continuity Theorem on the space of tempered distributions. ArXiv Mathematics e-prints (2017). arXiv: 1706.09326.

H. Biermé, O. Durieu and Y. Wang. Invariance principles for operator-scaling Gaussian random fields. Ann. Appl. Probab. 27 (2), 1190-1234 (2017). MR3655864.

H. Biermé and A. Estrade. Poisson random balls: self-similarity and x-ray images. Adv. in Appl. Probab. 38 (4), 853-872 (2006). MR2285684.

H. Biermé, A. Estrade and I. Kaj. Self-similar random fields and rescaled random balls models. J. Theoret. Probab. 23 (4), 1110-1141 (2010). MR2735739.

H. Biermé and C. Lacaux. Hölder regularity for operator scaling stable random fields. Stochastic Process. Appl. 119 (7), 2222-2248 (2009). MR2531090. 
H. Biermé, M. M. Meerschaert and H.-P. Scheffler. Operator scaling stable random fields. Stochastic Process. Appl. 117 (3), 312-332 (2007). MR2290879.

T. Bojdecki, L. G. Gorostiza and A. Talarczyk. A long range dependence stable process and an infinite variance branching system. Ann. Probab. 35 (2), 500-527 (2007). MR2308586.

J.-C. Breton and C. Dombry. Rescaled weighted random ball models and stable self-similar random fields. Stochastic Process. Appl. 119 (10), 3633-3652 (2009). MR2568289.

J.-C. Breton and C. Dombry. Functional macroscopic behavior of weighted random ball model. ALEA Lat. Am. J. Probab. Math. Stat. 8, 177-196 (2011). MR2786559.

J.-C. Breton and R. Gobard. Infinite dimensional functional convergences in random balls model. ESAIM Probab. Stat. 19, 782-793 (2015). MR3438258.

R. L. Dobrushin. Gaussian and their subordinated self-similar random generalized fields. Ann. Probab. 7 (1), 1-28 (1979). MR515810.

O. Durieu and Y. Wang. From random partitions to fractional Brownian sheets $(2018+)$. To appear in Bernoulli.

X. Fernique. Processus linéaires, processus généralisés. Ann. Inst. Fourier (Grenoble) 17 (fasc. 1), 1-92 (1967). MR0221576.

R. Gaigalas. A Poisson bridge between fractional Brownian motion and stable Lévy motion. Stochastic Process. Appl. 116 (3), 447-462 (2006). MR2199558.

B. Galerne. Computation of the perimeter of measurable sets via their covariogram. Applications to random sets. Image Anal. Stereol. 30 (1), 39-51 (2011). MR2816305.

I. M. Gel'fand and G. E. Shilov. Generalized functions. Vol. I: Properties and operations. Translated by Eugene Saletan. Academic Press, New York-London (1964). MR0166596.

I. M. Gel'fand and N. Y. Vilenkin. Generalized functions. Vol. 4: Applications of harmonic analysis. Translated by Amiel Feinstein. Academic Press, New York London, 1964 (1964). MR0173945.

R. Gobard. Random balls model with dependence. J. Math. Anal. Appl. 423 (2), 1284-1310 (2015). MR3278199.

M. Görgens and I. Kaj. Gaussian processes, bridges and membranes extracted from selfsimilar random fields. ArXiv Mathematics e-prints (2014). arXiv: 1410.0511.

R. A. Holley and D. W. Stroock. Generalized Ornstein-Uhlenbeck processes and infinite particle branching Brownian motions. Publ. Res. Inst. Math. Sci. 14 (3), 741-788 (1978). MR527199.

K. Itô. Distribution-valued processes arising from independent Brownian motions. Math. Z. 182 (1), 17-33 (1983). MR686883.

I. Kaj, L. Leskelä, I. Norros and V. Schmidt. Scaling limits for random fields with long-range dependence. Ann. Probab. 35 (2), 528-550 (2007). MR2308587.

I. Kaj and M. S. Taqqu. Convergence to fractional Brownian motion and to the Telecom process: the integral representation approach. In In and out of equilibrium. 2, volume 60 of Progr. Probab., pages 383-427. Birkhäuser, Basel (2008). MR2477392.

O. Kallenberg. Foundations of modern probability. Probability and its Applications (New York). Springer-Verlag, New York (1997). ISBN 0-387-94957-7. MR1464694. 
G. Kallianpur and J. Xiong. Stochastic differential equations in infinite-dimensional spaces, volume 26 of Institute of Mathematical Statistics Lecture NotesMonograph Series. Institute of Mathematical Statistics, Hayward, CA (1995). ISBN 0-940600-38-2. MR1465436.

C. Kipnis and C. Landim. Scaling limits of interacting particle systems, volume 320 of Grundlehren der Mathematischen Wissenschaften [Fundamental Principles of Mathematical Sciences]. Springer-Verlag, Berlin (1999). ISBN 3-540-64913-1. MR1707314.

F. Lavancier. Invariance principles for non-isotropic long memory random fields. Stat. Inference Stoch. Process. 10 (3), 255-282 (2007). MR2321311.

Y. Li, W. Wang and Y. Xiao. Exact moduli of continuity for operator-scaling Gaussian random fields. Bernoulli 21 (2), 930-956 (2015). MR3338652.

Y. Li and Y. Xiao. Occupation time fluctuations of weakly degenerate branching systems. J. Theoret. Probab. 25 (4), 1119-1152 (2012). MR2993016.

M. M. Meerschaert and H.-P. Scheffler. Limit theorems for continuous-time random walks with infinite mean waiting times. J. Appl. Probab. 41 (3), 623-638 (2004). MR2074812.

M. M. Meerschaert, W. Wang and Y. Xiao. Fernique-type inequalities and moduli of continuity for anisotropic Gaussian random fields. Trans. Amer. Math. Soc. 365 (2), 1081-1107 (2013). MR2995384.

T. Mikosch, S. Resnick, H. Rootzén and A. Stegeman. Is network traffic approximated by stable Lévy motion or fractional Brownian motion? Ann. Appl. Probab. 12 (1), 23-68 (2002). MR1890056.

V. Pilipauskaitè and D. Surgailis. Anisotropic scaling of the random grain model with application to network traffic. J. Appl. Probab. 53 (3), 857-879 (2016). MR3570099.

V. Pipiras and M. S. Taqqu. Long-range dependence and self-similarity. Cambridge Series in Statistical and Probabilistic Mathematics, [45]. Cambridge University Press, Cambridge (2017). ISBN 978-1-107-03946-9. MR3729426.

D. Puplinskaitè and D. Surgailis. Scaling transition for long-range dependent Gaussian random fields. Stochastic Process. Appl. 125 (6), 2256-2271 (2015). MR3322863.

G. Samorodnitsky. Stochastic processes and long range dependence. Springer Series in Operations Research and Financial Engineering. Springer, Cham (2016). MR3561100.

G. Samorodnitsky and M. S. Taqqu. Stable non-Gaussian random processes. Stochastic Modeling. Chapman \& Hall, New York (1994). ISBN 0-412-05171-0. MR1280932.

Y. Shen and Y. Wang. Operator-scaling Gaussian random fields via aggregation. ArXiv Mathematics e-prints (2017). arXiv: 1712.0708.

E. M. Stein. Harmonic analysis: real-variable methods, orthogonality, and oscillatory integrals, volume 43 of Princeton Mathematical Series. Princeton University Press, Princeton, NJ (1993). ISBN 0-691-03216-5. MR1232192.

M. Unser and P. D. Tafti. An introduction to sparse stochastic processes. Cambridge University Press, Cambridge (2014). ISBN 978-1-107-05854-5. MR3495257.

Y. Wang. An invariance principle for fractional Brownian sheets. J. Theoret. Probab. 27 (4), 1124-1139 (2014). MR3278934. 
Y. Xiao. Sample path properties of anisotropic Gaussian random fields. In $A$ minicourse on stochastic partial differential equations, volume 1962 of Lecture Notes in Math., pages 145-212. Springer, Berlin (2009). MR2508776. 